\title{
四氢呋喃螺氧化吲哚衍生物的一锅法高效合成
}

\author{
郭掀郭亚军 孔德志卢会杰华远照* 王敏灿* \\ (郑州大学化学学院 绿色催化研究所 郑州 450000)
}

\begin{abstract}
摘要 报道了 $\alpha$-羟基芳基酮和 $\beta, \gamma$-不饱和- $\alpha$-酮酰胺发生的 Michael/半缩酮化和傅-克(Friedel-Crafts)反应的两步一锅反 应. 该方法利用不包含氧化吲哚和四氢呋喃结构的链状底物, 高效构建出包含螺碳原子、氧化吲哚环和四氢呋喃环的 四氢呋喃螺氧化吲哚衍生物.

关键词一锅法; 串联反应; 四氢呋喃螺氧化吲哚
\end{abstract}

\section{Efficient Synthesis of Tetrahydrofuran Spirooxindoles via One-Pot Reaction}

\author{
Guo, Xin Guo, Yajun Kong, Dezhi Lu, Huijie Hua, Yuanzhao* Wang, Mincan* \\ (Institute of Green Catalysis, College of Chemistry, Zhengzhou University, Zhengzhou 450000)
}

\begin{abstract}
A one-pot reaction of Michael/hemiketalization and Fridel-Crafts reaction of $\alpha$-hydroxy aryl ketones and $\beta, \gamma$-unsaturated $\alpha$-ketoamides has been developed. The process enables efficient synthesis of tetrahydrofuran spirooxindoles using chain substrates that do not contain oxindole and tetrahydrofuran skeletons. A spiro-carbon center, an oxindole ring and a tetrahydrofuran ring, are constructed in this process.

Keywords one-pot; cascade reaction; tetrahydrofuran spirooxindoles
\end{abstract}

\section{Introduction}

Spirocyclic oxindoles are privileged structural motifs existing in a broad range of natural products and pharmaceuticals, which possess significant biological activities. ${ }^{[1]}$ It has been shown that substituents and spatial structures greatly influence the properties of those compounds. ${ }^{[2]}$ And potentially promising skeletons are those bearing a heteroatom (especially $\mathrm{N}$ or $\mathrm{O}$ ) in the spiro stereocenter at the $\mathrm{C}(3)$ position of the oxindole. ${ }^{[3]}$ Among various spirocyclic oxindoles, tetrahydrofuran spirooxindole derivatives have also attracted much attention of organic chemists for their potential biological activities. For example, spiro[furo-2,3'oxindole]s could inhibit the growth of lung adenocarcinoma (A549) cells and hepatocellular carcinoma (HepG2) cells. ${ }^{[4]}$ Consequently, a lot of elegant methods for their generation have been developed. ${ }^{[5]}$

Among these documented methods, there are two pathways to access tetrahydrofuran spirooxindoles. One method for the synthesis of tetrahydrofuran spirooxindoles starts with isatins or 3-hydroxy-2-oxindoles containing ring $\mathrm{A}$, followed by the construction of the spiro-carbon center and the formation of ring B (Pathway a, Scheme 1). ${ }^{[6]}$ The other needs readily available B-ring-containing amides to construct spiro-carbon and form ring A (Pathway b, Scheme 1). ${ }^{[7]}$ Only one ring is built and the other has already existed in one of the substrates in both above strategies. Meanwhile, sometimes these strategies have some inherent drawbacks, such as harsh reaction conditions, multiple operation procedures, poor compatibility of functional groups, and poor availability of the starting material to diversify the molecular structure. It is highly desirable and challenging to develop simple and integrated methods for the construction of a spiro-carbon center, ring $\mathrm{A}$, and ring $\mathrm{B}$ in one-pot reaction.

Recently, our group has endeavored to develop the applications of metal-catalysts. ${ }^{[8]}$ And we have successfully realized some dinuclear metal-catalytic asymmetric cascade reactions for the synthesis of structurally novel and functionalized compounds, ${ }^{[9]}$ such as Michael/hemiketalization to construct five- or six-membered oxygenated het-

\footnotetext{
* Corresponding authors. E-mail: hyzhao@zzu.edu.cn; wangmincan@zzu.edu.cn Received March 11, 2020; revised April 20, 2020; published online April 30, 2020.

Project supported by the National Natural Science Foundation of China (No. 21871237), the China Postdoctoral Science Foundation (No: 2017M622361) and the Education Department of Henan Province (Nos. 17B150014, 18B150028).

国家自然科学基金(No. 21272216)、中国博士后科学基金(No. 2017M622361)和河南省教育厅(Nos. 17B150014, 18B150028)资助项目.
} 


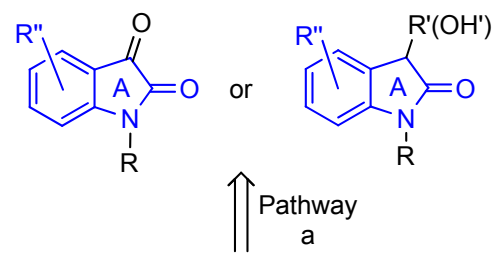<smiles>[R7]c1cccc2c1N([R])C(=O)C21CCCO1</smiles>

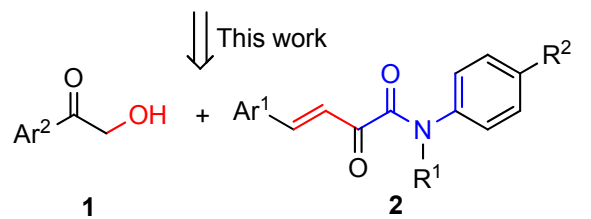

Scheme 1 Construction of tetrahydrofuran spirooxindoles

erocycles, ${ }^{\left[{ }^{[a-9 b]}\right.}$ phospha-Michael/Michael cascade reaction for the synthesis of 1,2,3-trisubstituted indanes, ${ }^{[9 \mathrm{c}]} \mathrm{Kno}-$ evenagel/Michael/cyclization for the synthesis of dihydrofuran spirooxindoles, ${ }^{[9 \mathrm{~d}]}$ Michael/transesterification for the formation of spiro-indanone $\gamma$-butyrolactones, ${ }^{\left[{ }^{[\mathrm{e}]}\right.}$ and asymmetric exo'-selective [3+2] cycloaddition to construct trifluoromethyl-substituted 2,3-pyrrolidinyl dispirooxindoles. ${ }^{[9 \mathrm{f}]}$ Particularly, we recently demonstrated that $\beta, \gamma$-unsaturated $\alpha$-ketoamides could serve as especially appropriate Michael acceptors for the enantioselective construction of dispirocyclic oxindoles through cascade Michael/cyclization/Friedel-Crafts reaction. ${ }^{[9 \mathrm{~g}-9 \mathrm{~h}]}$

As a continuation, we envisioned that the reaction of $\alpha$-hydroxy aryl ketones 1 with $\beta, \gamma$-unsaturated $\alpha$-ketoamides 2 may provide a straightforward approach to construct multisubstituted tetrahydrofuran spirooxindoles in one pot reaction. Herein we wish to report our progress with acceptable results in this area. ${ }^{[10]}$

\section{Results and discussion}

\subsection{Catalyst screening and optimization}

Initially, $\alpha$-hydroxy aryl ketone $\mathbf{1 a}$ and $\beta, \gamma$-unsaturated $\alpha$-ketoamide $2 \mathbf{a}$ were chosen as model substrates for optimization studies (Table 1). Reactions were conducted in dichloromethane (DCM) at room temperature for $24 \mathrm{~h}$. However, no obvious reaction occurred when $\mathrm{ZnCl}_{2}$ (10 mol\%) was used as catalyst (Table 1, Entry 1). A number of Lewis acids, such as $\mathrm{FeCl}_{3}, \mathrm{Zn}(\mathrm{OTf})_{2}, \mathrm{Zn}(\mathrm{OAc})_{2}$, $\mathrm{Cu}(\mathrm{OTf})_{2}, \mathrm{Cu}(\mathrm{OAc})_{2}, \mathrm{Mn}(\mathrm{OAc})_{2}, \mathrm{La}(\mathrm{OAc})_{2}$, and so on, were also tested, and none of them could catalyze the model reaction (Table 1, Entries 1 $\sim 7$ ). Then, some bases were employed as catalysts in this model reaction. Fortunately, the product 3 was obtained in $49 \%$ isolated yield when $\mathrm{NaOH}$ was used (Table 1, Entry 8). Encouraged by this, $\mathrm{KOH}$ and $\mathrm{CH}_{3} \mathrm{ONa}$ were examined subsequently, and the yields of product 3 were improved to $49 \%$ and $70 \%$, respectively (Table 1, Entries 9 and 10). A Brønsted base $\mathrm{ZnEt}_{2}$ was also evaluated, and gave the product 3 in $81 \%$ yield (Table 1, Entry 11). However, no reaction occurred when Lewis base $\mathrm{NEt}_{3}$ was used as catalyst (Table 1, Entry 12).

Table 1 Optimization of the reaction conditions ${ }^{a}$

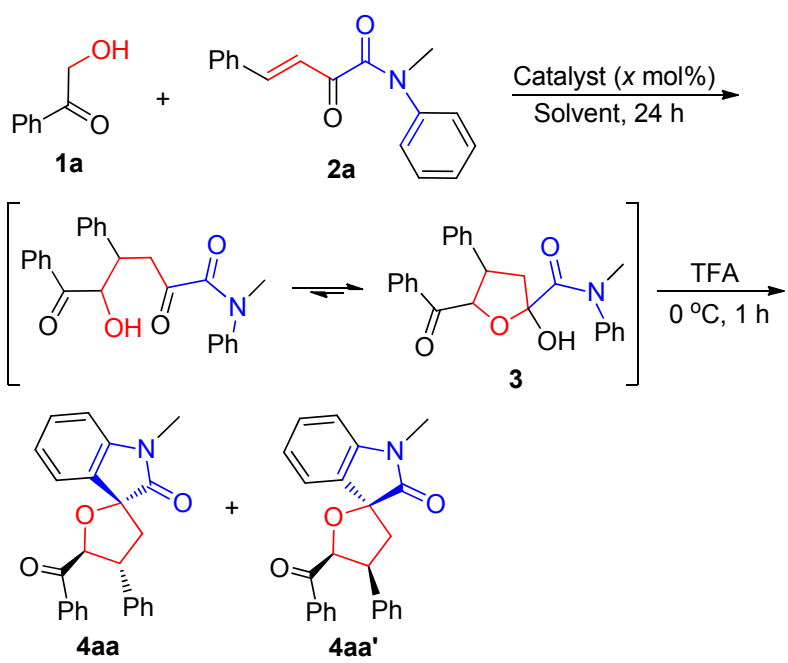

\begin{tabular}{clclcc}
\hline Entry & Catalyst & $x /$ mol\% & Solvent & $\begin{array}{c}\text { Yield/\% } \\
\text { of } 3\end{array}$ & $\begin{array}{c}\text { Yield/\% of } \\
\mathbf{4} / \mathbf{4}^{\prime}\left(\mathbf{4} / \mathbf{4}^{\prime}\right)\end{array}$ \\
\hline 1 & $\mathrm{ZnCl}_{2}$ & 10 & $\mathrm{CH}_{2} \mathrm{Cl}_{2}$ & Trace & - \\
2 & $\mathrm{FeCl}_{3}$ & 10 & $\mathrm{CH}_{2} \mathrm{Cl}_{2}$ & $\mathrm{NR}$ & - \\
3 & $\mathrm{Zn}(\mathrm{OTf})_{2}$ & 10 & $\mathrm{CH}_{2} \mathrm{Cl}_{2}$ & $\mathrm{NR}$ & - \\
4 & $\mathrm{Cu}(\mathrm{OTf})_{2}$ & 10 & $\mathrm{CH}_{2} \mathrm{Cl}_{2}$ & $\mathrm{NR}$ & - \\
5 & $\mathrm{La}(\mathrm{OAc})_{3}$ & 10 & $\mathrm{CH}_{2} \mathrm{Cl}_{2}$ & $\mathrm{NR}$ & - \\
6 & $\mathrm{Co}(\mathrm{OAc})_{2}$ & 10 & $\mathrm{CH}_{2} \mathrm{Cl}_{2}$ & $\mathrm{NR}$ & - \\
7 & ${\mathrm{Zn}(\mathrm{OAc})_{2}}_{10}$ & $\mathrm{CH}_{2} \mathrm{Cl}_{2}$ & $\mathrm{NR}$ & - \\
8 & $\mathrm{NaOH}_{9}$ & 10 & $\mathrm{CH}_{2} \mathrm{Cl}_{2}$ & 45 & - \\
9 & $\mathrm{KOH}_{10}$ & 10 & $\mathrm{CH}_{2} \mathrm{Cl}_{2}$ & 49 & - \\
11 & $\mathrm{CH}_{3} \mathrm{ONa}$ & 10 & $\mathrm{CH}_{2} \mathrm{Cl}_{2}$ & 70 & - \\
12 & $\mathrm{NEt}_{3}$ & 10 & $\mathrm{CH}_{2} \mathrm{Cl}_{2}$ & 81 & - \\
13 & $\mathrm{ZnEt}_{2}$ & 10 & $\mathrm{CH}_{2} \mathrm{Cl}_{2}$ & $\mathrm{NR}$ & - \\
14 & $\mathrm{ZnEt}_{2}$ & 10 & $\mathrm{THF}^{2}$ & 62 & - \\
15 & $\mathrm{ZnEt}_{2}$ & 10 & $\mathrm{Belluene}_{2}$ & 64 & - \\
16 & $\mathrm{ZnEt}_{2}$ & 10 & $\mathrm{CHCl}_{3}$ & 76 & - \\
17 & $\mathrm{ZnEt}_{2}$ & 10 & $\mathrm{CH}_{3} \mathrm{CN}_{2}$ & 79 & - \\
$18^{b}$ & $\mathrm{ZnEt}_{2}$ & 5 & $\mathrm{CH}_{2} \mathrm{Cl}_{2}$ & 76 & - \\
$19^{c}$ & $\mathrm{ZnEt}_{2}$ & 20 & $\mathrm{CH}_{2} \mathrm{Cl}_{2}$ & 88 & - \\
$20^{c}$ & $\mathrm{ZnEt}_{2}$ & 30 & $\mathrm{CH}_{2} \mathrm{Cl}_{2}$ & 88 & - \\
$21^{d, e}$ & $\mathrm{ZnEt}_{2}$ & 20 & $\mathrm{CH}_{2} \mathrm{Cl}_{2}$ & - & $60(32 / 28)$ \\
$22^{d, f}$ & $\mathrm{ZnEt}_{2}$ & 20 & $\mathrm{CH}_{2} \mathrm{Cl}_{2}$ & - & $71(39 / 32)$ \\
$23^{d, g}$ & $\mathrm{ZnEt}_{2}$ & 20 & $\mathrm{CH}_{2} \mathrm{Cl}_{2}$ & - & $84(46 / 38)$ \\
\hline
\end{tabular}

${ }^{a}$ Unless otherwise noted, all the reactions were conducted with $10 \mathrm{~mol} \%$ catalyst, $0.20 \mathrm{mmol}$ of $\mathbf{1}$, and $0.21 \mathrm{mmol}$ of $\mathbf{2}$ in $2 \mathrm{~mL}$ of solvent at $20{ }^{\circ} \mathrm{C}$ for $24 \mathrm{~h}^{b}{ }^{b}$ Reaction time was $40 \mathrm{~h} .{ }^{c}$ Reaction time was $6 \mathrm{~h}^{d}{ }^{d} 6 \mathrm{~h}$ later, acid was added at $0{ }^{\circ} \mathrm{C}$ and the mixture was stirred for another $1 \mathrm{~h} .{ }^{e}$ Two drops of $\mathrm{H}_{2} \mathrm{SO}_{4}$ was added. ${ }^{f} 0.5 \mathrm{~mL}$ of $\mathrm{HCl}$ was added. ${ }^{g} 0.5 \mathrm{~mL}$ of $\mathrm{CF}_{3} \mathrm{COOH}$ (TFA) was added.

Next, the evaluation of different solvents, including tetrahydrofuran (THF), toluene, benzene, $\mathrm{CHCl}_{3}$, and $\mathrm{CH}_{3} \mathrm{CN}$ indicated that $\mathrm{CH}_{2} \mathrm{Cl}_{2}$ was the optimal solvent which gave the product 3 in $81 \%$ yield (Table 1, Entries 11, 13 17). When the catalyst loading amount was lowered to $5 \mathrm{~mol} \%$, 
the yield of product 3 was decreased to $76 \%$ and the reaction time was prolonged to $48 \mathrm{~h}$. Once increasing the catalyst loading amount to $20 \mathrm{~mol} \%$, the yield of product 3 was increased to $88 \%$ in $6 \mathrm{~h}$. While the yield was not further improved when $30 \mathrm{~mol} \%$ catalyst loading was employed, which indicated that $20 \mathrm{~mol}^{\%}$ of $\mathrm{ZnEt}_{2}$ was the best choice (Table 1, Entries 18 20).

The ${ }^{1} \mathrm{H}$ NMR and ${ }^{13} \mathrm{C}$ NMR spectra revealed that product 3 was obtained as a mixture of the chain structure (Michael addition product) and the cyclic structure (Michael/hemiketalization product). ${ }^{[10]}$ There was a dynamic balance between the chain structure and the cyclic structure, which led to the difficulty to purify and characterize. It was found that this mixture could give the title compound tetrahydrofuran spirooxindole 4/4' through an intramolecular Friedel-Crafts reaction, when it was treated with $\mathrm{H}_{2} \mathrm{SO}_{4}$ (Table 1, Entry 21). Then, concentrated hydrochloric acid $(\mathrm{HCl})$ and $\mathrm{CF}_{3} \mathrm{COOH}$ (TFA) were evaluated, and TFA was the optimal choice giving product $\mathbf{4} / \mathbf{4}^{\prime}$ in $84 \%$ total yields through 2 steps one-pot reaction (Table 1, Entries 22 and 23). Although the diastereoselectivity was low, the two isomers of anti-4aa and syn-4aa' could be separated by flash column chromatography on silica gel.

Thus the optimal conditions were determined as follows (Table 1, Entry 23): $20 \mathrm{~mol} \% \mathrm{ZnEt}_{2}, 2 \mathrm{~mL}$ of $\mathrm{CH}_{2} \mathrm{Cl}_{2}$, $\alpha$-hydroxy aryl ketones $1(0.20 \mathrm{mmol}), \beta, \gamma$-unsaturated $\alpha$-ketoamide $2(0.21 \mathrm{mmol})$ at $20{ }^{\circ} \mathrm{C}$ for $6 \mathrm{~h}$. Then $0.5 \mathrm{~mL}$ of TFA was dropwise added at $0{ }^{\circ} \mathrm{C}$ and stirred for another $1 \mathrm{~h}$.

\subsection{Substrate scope}

The substrate scope of this multisubstituted tetrahydrofuran spirooxindoles forming reaction was explored under the optimal conditions. Using $\mathbf{1 a}$ as a model substrate, the $N$-protecting groups $\mathrm{R}^{1}$ of $\beta, \gamma$-unsaturated- $\alpha$-ketoamides 2 was first examined. The experiment results revealed that the steric bulk of the substituent at $N$ atom in $\mathbf{2 a} \sim \mathbf{2} \mathbf{c}$ had an obvious influence on the yield in this reaction. When substituent $\mathrm{R}^{1}$ was changed from Me to Et or Bn, the total yields of products $4 / 4^{\prime}$ decreased to $53 \%$ and $51 \%$ (Table 2, Entries 2 and 3 ). The reason might owe to the larger steric hindrance of Et or Bn.

Then a series of $N$-methyl $\beta, \gamma$-unsaturated- $\alpha$-ketoamides 2 bearing various substituents attached on the phenyl ring were investigated. Substrates $\mathbf{2 d} \sim \mathbf{2 h}$ with electron-withdrawing halogen groups $(\mathrm{F}, \mathrm{Cl}$ or $\mathrm{Br}$ ) or electron-donating groups (Me or $\mathrm{MeO}$ ) at the para-position of the $N$-aromatic ring reacted with $\mathbf{1 a}$ efficiently under the standard conditions. And the corresponding products $4 / \mathbf{4}^{\prime}$ were obtained in $62 \% \sim 87 \%$ total yields (Table 2 , Entries $4 \sim 8$ ).

The effect of the para-substituent of the phenyl ring $\left(\mathrm{Ar}^{2}\right)$ was also explored. Various functional groups, both the electron-withdrawing groups $\left(\mathrm{NO}_{2}, \mathrm{~F}, \mathrm{Cl}\right.$ or $\left.\mathrm{Br}\right)$ and the electron-donating groups ( $\mathrm{Me}$ or $\mathrm{MeO})$, were well tolerated (Table 2, Entries 9 14). Meanwhile, it was also observed that nitro group of $\mathrm{Ar}^{2}$ affected the yield obviously (51\%, Table 2, Entry 9). Substrates 20 and 2p with Br substituent at the meta- or ortho-position of $\mathrm{Ar}^{2}$ provided the corresponding products $\mathbf{4} / \mathbf{4}^{\prime}$ in $72 \%$ and $79 \%$ total yields, respectively (Table 2, Entries 15, 16). Notably, 1-naphthyl and 2-naphthyl substituted ketoamides $\mathbf{2 q} \sim \mathbf{2 r}$ took part in this reaction successfully, giving corresponding products $4 / 4^{\prime}$ in total yields of $75 \%$ and $84 \%$, respectively. Further study showed that 2-furyl substituted ketoamide $2 \mathrm{~s}$ was also a suitable substrate to react with $\mathbf{1 a}$ in $85 \%$ total yields.

The reaction of ketoamide $\mathbf{2 a}$ with various nucleophiles was next investigated. A series of nucleophiles $\mathbf{1 b} \sim \mathbf{1 i}$ bearing either electron-withdrawing groups ( $\mathrm{F}, \mathrm{Cl}$ and $\mathrm{Br}$ ) or electron-donating groups $(\mathrm{Me}$ and $\mathrm{MeO}$ ) at aromatic ring $\mathrm{Ar}^{1}$ underwent the reaction efficiently, giving $71 \% \sim 95 \%$ total yields (Table 2, Entries 20 27).

To determine the relative configuration of the products, product 4al' was treated with hydroxylamine hydrochloride and $\mathrm{NaOAc}$ in $\mathrm{CH}_{3} \mathrm{OH}$, which led to the corresponding product of oxime $\mathbf{5}^{\prime}$ in $96 \%$ yield (Scheme 2). A single crystal of compound $\mathbf{5}^{\prime}$ was obtained for X-ray crystallographic analysis (Figure 1). ${ }^{[1]}$ Thus, the relative stereochemistry of 4al' could be deduced.
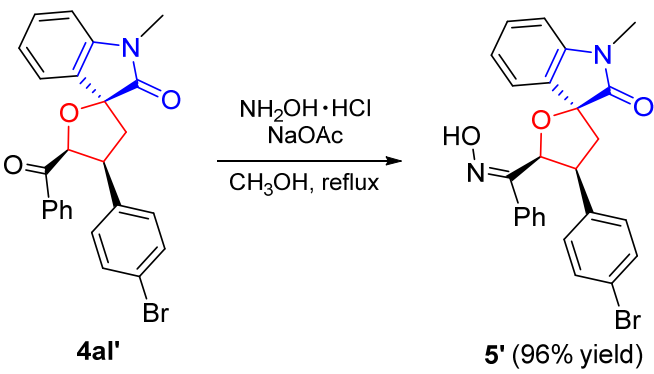

Scheme 2 Derivatization of product 4al'

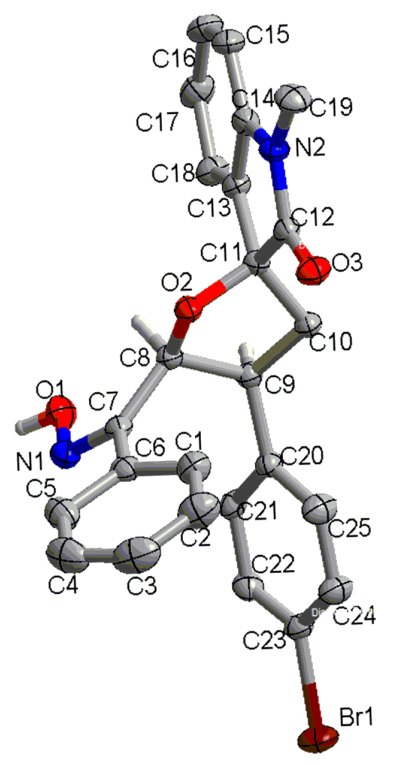

Figure 1 X-ray structure of compound 5'

\section{Conclusions}

In conclusion, we have developed an efficient method for the direct formation of tetrahydrofuran spirooxindoles, 
Table 2 Substrate scope of the reaction ${ }^{a}$

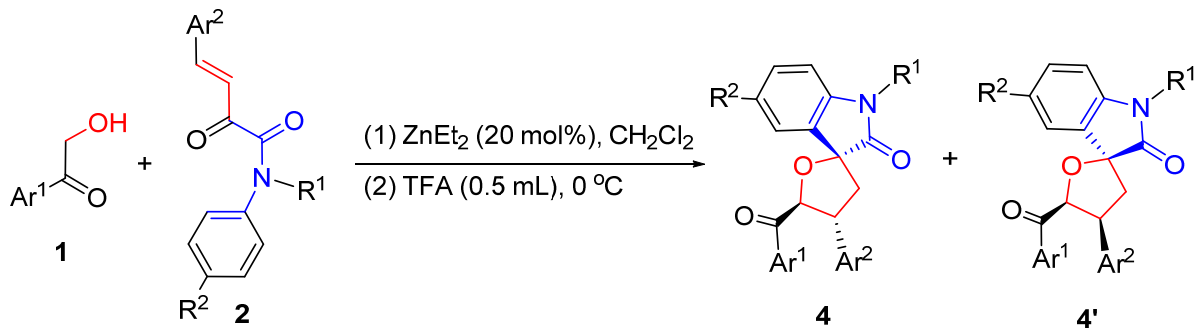

\begin{tabular}{|c|c|c|c|c|c|c|}
\hline Entry & $\mathrm{Ar}^{1}$ & $\mathrm{R}^{1}$ & $\mathrm{Ar}^{2}$ & $\mathrm{R}^{2}$ & Product & Yield/\% (4/4') \\
\hline 1 & $\mathrm{Ph}$ & $\mathrm{Me}$ & $\mathrm{Ph}$ & $\mathrm{H}$ & 4aa/4aa' & $84(46 / 38)$ \\
\hline 2 & $\mathrm{Ph}$ & $\mathrm{Et}$ & $\mathrm{Ph}$ & $\mathrm{H}$ & $4 a b / 4 a b^{\prime}$ & $53(22 / 31)$ \\
\hline 3 & $\mathrm{Ph}$ & $\mathrm{Bn}$ & $\mathrm{Ph}$ & $\mathrm{H}$ & 4ac/4ac' & $51(25 / 26)$ \\
\hline 4 & $\mathrm{Ph}$ & $\mathrm{Me}$ & $\mathrm{Ph}$ & $\mathrm{F}$ & 4ad/4ad' & $86(56 / 30)$ \\
\hline 5 & $\mathrm{Ph}$ & $\mathrm{Me}$ & $\mathrm{Ph}$ & $\mathrm{Cl}$ & $4 a e / 4 a e^{\prime}$ & $87(57 / 30)$ \\
\hline 6 & $\mathrm{Ph}$ & $\mathrm{Me}$ & $\mathrm{Ph}$ & $\mathrm{Br}$ & 4af/4af' & $61(39 / 22)$ \\
\hline 7 & $\mathrm{Ph}$ & $\mathrm{Me}$ & $\mathrm{Ph}$ & $\mathrm{Me}$ & 4ag/4ag' & $74(44 / 30)$ \\
\hline 8 & $\mathrm{Ph}$ & $\mathrm{Me}$ & $\mathrm{Ph}$ & $\mathrm{MeO}$ & 4ah/4ah' & $76(37 / 39)$ \\
\hline 9 & $\mathrm{Ph}$ & $\mathrm{Me}$ & $4-\mathrm{O}_{2} \mathrm{NC}_{6} \mathrm{H}_{4}$ & $\mathrm{H}$ & 4ai/4ai' & $51(24 / 27)$ \\
\hline 10 & $\mathrm{Ph}$ & $\mathrm{Me}$ & $4-\mathrm{FC}_{6} \mathrm{H}_{4}$ & $\mathrm{H}$ & 4aj/4aj' & $77(39 / 38)$ \\
\hline 11 & $\mathrm{Ph}$ & $\mathrm{Me}$ & $4-\mathrm{ClC}_{6} \mathrm{H}_{4}$ & $\mathrm{H}$ & 4ak/4ak' & $88(44 / 44)$ \\
\hline 12 & $\mathrm{Ph}$ & $\mathrm{Me}$ & $4-\mathrm{BrC}_{6} \mathrm{H}_{4}$ & $\mathrm{H}$ & 4al/4al' & $94(61 / 33)$ \\
\hline 13 & $\mathrm{Ph}$ & $\mathrm{Me}$ & 4- $\mathrm{MeC}_{6} \mathrm{H}_{4}$ & $\mathrm{H}$ & 4am/4am' & $79(41 / 38)$ \\
\hline 14 & $\mathrm{Ph}$ & $\mathrm{Me}$ & 4- $\mathrm{MeOC}_{6} \mathrm{H}_{4}$ & $\mathrm{H}$ & 4an/4an' & $72(39 / 33)$ \\
\hline 15 & $\mathrm{Ph}$ & $\mathrm{Me}$ & $3-\mathrm{BrC}_{6} \mathrm{H}_{4}$ & $\mathrm{H}$ & 4ao/4ao' & $72(49 / 23)$ \\
\hline 16 & $\mathrm{Ph}$ & $\mathrm{Me}$ & $2-\mathrm{BrC}_{6} \mathrm{H}_{4}$ & $\mathrm{H}$ & 4ap/4ap' & $79(50 / 29)$ \\
\hline 17 & $\mathrm{Ph}$ & $\mathrm{Me}$ & 1-Naphthyl & $\mathrm{H}$ & $4 \mathrm{aq} / 4 \mathrm{aq}^{\prime}$ & $75(37 / 38)$ \\
\hline 18 & $\mathrm{Ph}$ & $\mathrm{Me}$ & 2-Naphthyl & $\mathrm{H}$ & 4ar/4ar' & $84(58 / 26)$ \\
\hline 19 & $\mathrm{Ph}$ & $\mathrm{Me}$ & 2-Furyl & $\mathrm{H}$ & 4as/4as' & $85(43 / 42)$ \\
\hline 20 & $4-\mathrm{FC}_{6} \mathrm{H}_{4}$ & $\mathrm{Me}$ & $\mathrm{Ph}$ & $\mathrm{H}$ & $4 \mathrm{ba} / 4 \mathrm{ba}^{\prime}$ & $87(47 / 40)$ \\
\hline 21 & $4-\mathrm{ClC}_{6} \mathrm{H}_{4}$ & $\mathrm{Me}$ & $\mathrm{Ph}$ & $\mathrm{H}$ & $4 \mathrm{ca} / 4 \mathrm{ca}^{\prime}$ & $92(48 / 44)$ \\
\hline 22 & 4- $\mathrm{BrC}_{6} \mathrm{H}_{4}$ & $\mathrm{Me}$ & $\mathrm{Ph}$ & $\mathrm{H}$ & 4da/4da' & $94(59 / 36)$ \\
\hline 23 & 4- $\mathrm{MeC}_{6} \mathrm{H}_{4}$ & $\mathrm{Me}$ & $\mathrm{Ph}$ & $\mathrm{H}$ & $4 \mathrm{ea} / 4 \mathrm{ea}$ & $98(46 / 42)$ \\
\hline 24 & $4-\mathrm{MeOC}_{6} \mathrm{H}_{4}$ & $\mathrm{Me}$ & $\mathrm{Ph}$ & $\mathrm{H}$ & $4 \mathrm{fa} / \mathbf{4 f a}$ & $91(62 / 29)$ \\
\hline 25 & $3-\mathrm{BrC}_{6} \mathrm{H}_{4}$ & $\mathrm{Me}$ & $\mathrm{Ph}$ & $\mathrm{H}$ & 4ga/4ga' & $80(48 / 42)$ \\
\hline 26 & $3-\mathrm{MeOC}_{6} \mathrm{H}_{4}$ & $\mathrm{Me}$ & $\mathrm{Ph}$ & $\mathrm{H}$ & 4ha/4ha' & $78(41 / 37)$ \\
\hline 27 & $2-\mathrm{MeOC}_{6} \mathrm{H}_{4}$ & $\mathrm{Me}$ & $\mathrm{Ph}$ & $\mathrm{H}$ & $4 i a / 4 i a^{\prime}$ & $71(42 / 29)$ \\
\hline
\end{tabular}

${ }^{a}$ Unless otherwise noted, all the reactions were conducted with $20 \mathrm{~mol} \%$ catalyst $0.20 \mathrm{mmol}$ of $\mathbf{1}$, and $0.21 \mathrm{mmol}^{\circ} 2 \mathrm{in} 2 \mathrm{~mL}$ of $\mathrm{CH}_{2} \mathrm{Cl}_{2}$ at $20{ }^{\circ} \mathrm{C} .6 \mathrm{~h} \mathrm{later,} 0.5 \mathrm{~mL}$ of TFA was dropwise added at $0{ }^{\circ} \mathrm{C}$.

including the constructions of a spiro-carbon center, an oxindole ring, and a tetrahydrofuran ring in one-pot reaction. This chemistry is based on Michael/hemiketalization and Fridel-Crafts reaction of $\alpha$-hydroxy aryl ketones and $\beta, \gamma$-unsaturated $\alpha$-ketoamides. Using $\mathrm{ZnEt}_{2}$ and TFA as catalysts, the one-pot reaction proceeded efficiently and delivered a wide range of tetrahydrofuran spirooxindoles in moderate to good yields. We expect that this novel protocol would provide a convenient and versatile synthetic approach toward structurally diverse tetrahydrofuran spirooxindoles using simple chain substrates.

\section{Experimental section}

\subsection{General procedures}

The melting point (m.p.) was determined on an electrothermal digital melting point apparatus and uncorrected.
NMR spectra were recorded on an NMR spectrometer using $\mathrm{CDCl}_{3}$ or DMSO as the solvents and TMS as an internal standard (400 MHz for ${ }^{1} \mathrm{H} \mathrm{NMR}, 100 \mathrm{MHz}$ for ${ }^{13} \mathrm{C} \mathrm{NMR}$ ). HRMS was performed on a Q-TOF Micro LC/MS System ESI spectrometer.

\subsection{General procedure for the catalytic reaction}

In a flame-dried Schlenk tube, a solution of diethylzinc $(0.04 \mathrm{~mL}, 1.0 \mathrm{~mol} / \mathrm{L}$ in hexane, $0.04 \mathrm{mmol})$ was added to a solution of compound $1(0.2 \mathrm{mmol})$ and substrate $2(0.21$ $\mathrm{mmol})$ in dry $\mathrm{CH}_{2} \mathrm{Cl}_{2}(2 \mathrm{~mL})$ under nitrogen at $20{ }^{\circ} \mathrm{C}$. The mixture was stirred for $6 \mathrm{~h}$ to complete the Michael/ hemiketalization reaction (step 1 , detected by TLC). Then, $0.5 \mathrm{~mL}$ of TFA was added dropwise to the mixture at $0{ }^{\circ} \mathrm{C}$, and stirred for another $1 \mathrm{~h}$ at room temperature. Finally, the solution was diluted with a saturated solution of $\mathrm{Na}_{2} \mathrm{CO}_{3}$ and extracted with $\mathrm{CH}_{2} \mathrm{Cl}_{2}(10 \mathrm{~mL} \times 3)$. The combined 
organic layer was dried over $\mathrm{MgSO}_{4}$ and concentrated in vacuo. The residue was purified by flash column chromatography on silica gel to give products $\mathbf{4} \mathbf{a a}^{[10]}$ and $\mathbf{4 a} \mathbf{a}^{\prime}$, respectively.

5-Benzoyl-1'-methyl-4-phenyl-4,5-dihydro-3 $H$-spiro[furan-2,3'-indolin]-2'-one (4aa'): White solid (38\% yield), m.p. $209 \sim 211{ }^{\circ} \mathrm{C} ;{ }^{1} \mathrm{H}$ NMR $\left(400 \mathrm{MHz}, \mathrm{CDCl}_{3}\right) \delta: 7.70(\mathrm{~d}$, $J=7.6 \mathrm{~Hz}, 2 \mathrm{H}), 7.51(\mathrm{~d}, J=7.3 \mathrm{~Hz}, 1 \mathrm{H}), 7.45 \sim 7.25(\mathrm{~m}$, $6 \mathrm{H}), 7.18 \sim 7.00(\mathrm{~m}, 4 \mathrm{H}), 6.86(\mathrm{~d}, J=7.8 \mathrm{~Hz}, 1 \mathrm{H}), 5.97(\mathrm{~d}$, $J=8.2 \mathrm{~Hz}, 1 \mathrm{H}), 4.38$ (q, $J=8.3 \mathrm{~Hz}, 1 \mathrm{H}), 3.23$ (s, 3H), 3.08 (dd, $J=13.0,8.7 \mathrm{~Hz}, 1 \mathrm{H}), 2.69$ (dd, $J=12.9,8.2 \mathrm{~Hz}, 1 \mathrm{H})$; ${ }^{13} \mathrm{C} \mathrm{NMR}\left(101 \mathrm{MHz}, \mathrm{CDCl}_{3}\right) \delta: 195.9,174.7,143.55,138.6$, $136.5,132.8,131.5,130.1,128.7,128.4,128.3,128.2$, 127.1, 123.4, 123.0, 108.7, 85.2, 83.6, 49.0, 41.9, 26.5; HRMS (ESI) calcd for $\mathrm{C}_{25} \mathrm{H}_{22} \mathrm{NO}_{3}[\mathrm{M}+\mathrm{H}]^{+}$384.1594, found 384.1596.

5-Benzoyl-1'-ethyl-4-phenyl-4,5-dihydro-3H-spiro[furan2,3'-indolin]-2'-one (4ab'): White solid (31\% yield), m.p. 200 $202{ }^{\circ} \mathrm{C} ;{ }^{1} \mathrm{H}$ NMR (400 MHz, $\left.\mathrm{CDCl}_{3}\right) \delta: 7.69$ (d, $J=$ $7.7 \mathrm{~Hz}, 2 \mathrm{H}), 7.52(\mathrm{~d}, J=7.3 \mathrm{~Hz}, 1 \mathrm{H}), 7.45 \sim 7.22(\mathrm{~m}, 6 \mathrm{H})$, $7.17 \sim 6.97(\mathrm{~m}, 4 \mathrm{H}), 6.87(\mathrm{~d}, J=7.8 \mathrm{~Hz}, 1 \mathrm{H}), 5.98(\mathrm{~d}, J=$ $8.2 \mathrm{~Hz}, 1 \mathrm{H}), 4.37$ (q, $J=8.3 \mathrm{~Hz}, 1 \mathrm{H}), 3.78(\mathrm{q}, J=7.1 \mathrm{~Hz}$, 2H), 3.08 (dd, $J=12.8,8.7 \mathrm{~Hz}, 1 \mathrm{H}), 2.68$ (dd, $J=12.9,8.3$ $\mathrm{Hz}, 1 \mathrm{H}), 1.29(\mathrm{t}, J=7.2 \mathrm{~Hz}, 3 \mathrm{H}) ;{ }^{13} \mathrm{C} \mathrm{NMR}(101 \mathrm{MHz}$, $\left.\mathrm{CDCl}_{3}\right) \delta: 196.3,174.7,143.0,138.9,137.0,133.1,132.2$, $130.3,129.0,128.8,128.6,128.6,127.4,123.9,123.2$, 109.2, 85.6, 84.0, 49.3, 42.5, 35.5, 13.0; HRMS (ESI) calcd for $\mathrm{C}_{26} \mathrm{H}_{24} \mathrm{NO}_{3}[\mathrm{M}+\mathrm{H}]^{+}$398.1751, found 398.1754.

5-Benzoyl-1'-benzyl-4-phenyl-4,5-dihydro-3 $\mathrm{H}$-spiro[furan-2,3'-indolin]-2'-one (4ac'): White solid (26\% yield), m.p. $175 \sim 177{ }^{\circ} \mathrm{C} ;{ }^{1} \mathrm{H}$ NMR $\left(400 \mathrm{MHz}, \mathrm{CDCl}_{3}\right) \delta: 7.73(\mathrm{~d}$, $J=7.6 \mathrm{~Hz}, 2 \mathrm{H}), 7.54(\mathrm{~d}, J=7.2 \mathrm{~Hz}, 1 \mathrm{H}), 7.48 \sim 7.41(\mathrm{~m}$, $1 \mathrm{H}), 7.38 \sim 7.21(\mathrm{~m}, 10 \mathrm{H}), 7.19 \sim 7.04(\mathrm{~m}, 4 \mathrm{H}), 6.77(\mathrm{~d}$, $J=7.8 \mathrm{~Hz}, 1 \mathrm{H}), 6.03(\mathrm{~d}, J=8.3 \mathrm{~Hz}, 1 \mathrm{H}), 4.95(\mathrm{dd}, J=54.7$, $15.7 \mathrm{~Hz}, 2 \mathrm{H}), 4.43$ (q, $J=8.4 \mathrm{~Hz}, 1 \mathrm{H}), 3.19(\mathrm{dd}, J=12.8$, $9.0 \mathrm{~Hz}, 1 \mathrm{H}), 2.74(\mathrm{dd}, J=12.8,8.2 \mathrm{~Hz}, 1 \mathrm{H}) ;{ }^{13} \mathrm{C} \mathrm{NMR}(101$ $\left.\mathrm{MHz}, \mathrm{CDCl}_{3}\right) \delta: 196.3,175.4,142.9,138.7,136.9,135.9$, $133.1,131.9,130.3,129.2,129.0,128.8,128.6,128.5$, $128.1,127.8,127.5,123.8,123.4,110.1,85.5,84.1,49.4$, 44.5, 42.4; HRMS (ESI) calcd for $\mathrm{C}_{31} \mathrm{H}_{26} \mathrm{NO}_{3}[\mathrm{M}+\mathrm{H}]^{+}$ 460.1907, found 460.1909.

5-Benzoyl-5'-fluoro-1'-methyl-4-phenyl-4,5-dihydro-3 $H$ spiro[furan-2,3'-indolin]-2'-one (4ad'): White solid $(30 \%$ yield), m.p. $196 \sim 198{ }^{\circ} \mathrm{C}$; ${ }^{1} \mathrm{H}$ NMR (400 MHz, $\left.\mathrm{CDCl}_{3}\right) \delta$ : $7.67(\mathrm{~d}, J=7.7 \mathrm{~Hz}, 2 \mathrm{H}), 7.50 \sim 7.36(\mathrm{~m}, 1 \mathrm{H}), 7.35 \sim 7.21$ $(\mathrm{m}, 5 \mathrm{H}), 7.19 \sim 7.01(\mathrm{~m}, 4 \mathrm{H}), 6.88 \sim 6.71(\mathrm{~m}, 1 \mathrm{H}), 5.94(\mathrm{~d}$, $J=8.3 \mathrm{~Hz}, 1 \mathrm{H}), 4.33$ (dd, $J=16.8,8.4 \mathrm{~Hz}, 1 \mathrm{H}), 3.21$ (s, $3 \mathrm{H}), 3.10(\mathrm{dd}, J=12.8,9.1 \mathrm{~Hz}, 1 \mathrm{H}), 2.65(\mathrm{dd}, J=12.9,8.1$ $\mathrm{Hz}, 1 \mathrm{H}) ;{ }^{13} \mathrm{C}$ NMR $\left(101 \mathrm{MHz}, \mathrm{CDCl}_{3}\right) \delta: 195.6,174.5$, $159.3(\mathrm{~d}, J=240.3 \mathrm{~Hz}), 139.48(\mathrm{~d}, J=1.9 \mathrm{~Hz}), 138.0$, $136.5,132.8,128.6,128.4,128.3,128.2,127.2,116.1$, (d, $J=23.2 \mathrm{~Hz}), 111.7(\mathrm{~d}, J=24.7 \mathrm{~Hz}), 109.2(\mathrm{~d}, J=7.87 \mathrm{~Hz})$, 84.98, 83.7, 48.9, 41.8, 26.6; HRMS (ESI) calcd for $\mathrm{C}_{25} \mathrm{H}_{21} \mathrm{FNO}_{3}[\mathrm{M}+\mathrm{H}]^{+}$402.1500, found 402.1503.

5-Benzoyl-5'-chloro-1'-methyl-4-phenyl-4,5-dihydro-3Hspiro[furan-2,3'-indolin]-2'-one (4ae'): White solid $(30 \%$ yield), m.p. $180 \sim 182{ }^{\circ} \mathrm{C} ;{ }^{1} \mathrm{H}$ NMR $\left(600 \mathrm{MHz}, \mathrm{CDCl}_{3}\right) \delta$ :
$7.71 \sim 7.70(\mathrm{~m}, 2 \mathrm{H}), 7.56(\mathrm{~d}, J=7.7 \mathrm{~Hz}, 2 \mathrm{H}), 7.41 \sim 7.34$ $(\mathrm{m}, 2 \mathrm{H}), 7.31 \sim 7.26(\mathrm{~m}, 3 \mathrm{H}), 7.21 \sim 7.12(\mathrm{~m}, 2 \mathrm{H}), 6.96 \sim$ $6.82(\mathrm{~m}, 2 \mathrm{H}), 6.16(\mathrm{~d}, J=8.5 \mathrm{~Hz}, 1 \mathrm{H}), 4.89(\mathrm{dt}, J=10.5,8.2$ $\mathrm{Hz}, 1 \mathrm{H}), 3.23$ (s, 3H), $3.16(\mathrm{dd}, J=20.6,8.3 \mathrm{~Hz}, 2 \mathrm{H}), 2.52$ $(\mathrm{dd}, J=12.4,7.3 \mathrm{~Hz}, 1 \mathrm{H}) ;{ }^{13} \mathrm{C} \mathrm{NMR}\left(151 \mathrm{MHz}, \mathrm{CDCl}_{3}\right) \delta$ : $196.4,174.7,143.4,136.7,136.2,132.8,132.3,131.2$, $130.0,129.7,128.7,128.3,128.2,127.7,125.3,123.4$, 123.1, 108.6, 83.4, 81.9, 47.4, 40.6, 29.7, 26.5; HRMS (ESI) calcd for $\mathrm{C}_{25} \mathrm{H}_{21} \mathrm{ClNO}_{3}[\mathrm{M}+\mathrm{H}]^{+}$418.1204, found 418.1205 .

5-Benzoyl-5'-bromo-1'-methyl-4-phenyl-4,5-dihydro-3 $H$ spiro[furan-2,3'-indolin]-2'-one (4af'): White solid (23\% yield), m.p. $203 \sim 205{ }^{\circ} \mathrm{C}$; ${ }^{1} \mathrm{H}$ NMR (400 $\left.\mathrm{MHz}, \mathrm{CDCl}_{3}\right) \delta$ : $7.57 \sim 7.40(\mathrm{~m}, 3 \mathrm{H}), 7.36 \sim 7.28(\mathrm{~m}, 1 \mathrm{H}), 7.28 \sim 7.18(\mathrm{~m}$, $1 \mathrm{H}), 7.17 \sim 7.03(\mathrm{~m}, 4 \mathrm{H}), 6.95 \sim 6.80(\mathrm{~m}, 3 \mathrm{H}), 6.62(\mathrm{~d}, J=$ $8.3 \mathrm{~Hz}, 1 \mathrm{H}), 5.84(\mathrm{~d}, J=8.4 \mathrm{~Hz}, 1 \mathrm{H}), 4.31 \sim 4.16(\mathrm{~m}, 1 \mathrm{H})$, $3.03(\mathrm{~s}, 3 \mathrm{H}), 2.85$ (dd, $J=12.9,9.1 \mathrm{~Hz}, 1 \mathrm{H}), 2.49$ (dd, $J=$ 13.0, 8.3 Hz, 1H); ${ }^{13} \mathrm{C}$ NMR (101 MHz, $\left.\mathrm{CDCl}_{3}\right) \delta: 196.2$, $174.5,142.2,137.97,136.3,133.3,132.7,132.6,128.4$, $128.1,127.97,127.95,126.9,126.5,115.5,110.1,84.8$, 83.5, 48.6, 41.9, 26.1; HRMS (ESI) calcd for $\mathrm{C}_{25} \mathrm{H}_{21} \mathrm{BrN}-$ $\mathrm{O}_{3}[\mathrm{M}+\mathrm{H}]^{+}$462.0699, found 462.0700.

5-Benzoyl-1',5'-dimethyl-4-phenyl-4,5-dihydro-3 $H$ spiro[furan-2,3'-indolin]-2'-one (4ag'): White solid (30\% yield), m.p. 201 $203{ }^{\circ} \mathrm{C}$; ${ }^{1} \mathrm{H}$ NMR (400 MHz, $\left.\mathrm{CDCl}_{3}\right) \delta$ : $7.71(\mathrm{~d}, J=7.6 \mathrm{~Hz}, 2 \mathrm{H}), 7.47 \sim 7.36(\mathrm{~m}, 1 \mathrm{H}), 7.35 \sim 7.22$ $(\mathrm{m}, 5 \mathrm{H}), 7.20 \sim 7.02(\mathrm{~m}, 4 \mathrm{H}), 6.73(\mathrm{~d}, J=7.9 \mathrm{~Hz}, 1 \mathrm{H}), 5.95$ (d, $J=8.2 \mathrm{~Hz}, 1 \mathrm{H}), 4.38$ (q, $J=8.4 \mathrm{~Hz}, 1 \mathrm{H}), 3.19$ (s, 3H), 3.07 (dd, $J=12.9,8.7 \mathrm{~Hz}, 1 \mathrm{H}), 2.67$ (dd, $J=12.9,8.3 \mathrm{~Hz}$, $1 \mathrm{H}), 2.38(\mathrm{~s}, 3 \mathrm{H}) ;{ }^{13} \mathrm{C} \mathrm{NMR}\left(101 \mathrm{MHz}, \mathrm{CDCl}_{3}\right) \delta: 195.98$, $174.7,141.2,138.6,136.6,132.7,132.6,131.5,130.2$, $128.7,128.5,128.2,128.2,127.0,124.2,108.4,85.3,83.8$, 48.99, 41.9, 26.5, 21.2; HRMS (ESI) calcd for $\mathrm{C}_{26} \mathrm{H}_{24} \mathrm{NO}_{3}$ $[\mathrm{M}+\mathrm{H}]^{+}$398.1751, found 398.1753.

5-Benzoyl-5'-methoxy-1'-methyl-4-phenyl-4,5-dihydro3H-spiro[furan-2,3'-indolin]-2'-one (4ah'): White solid (39\% yield), m.p. $215 \sim 217{ }^{\circ} \mathrm{C} ;{ }^{1} \mathrm{H}$ NMR (400 MHz, $\left.\mathrm{CDCl}_{3}\right) \delta: 7.69(\mathrm{~d}, J=7.6 \mathrm{~Hz}, 2 \mathrm{H}), 7.45 \sim 7.35(\mathrm{~m}, 1 \mathrm{H})$, $7.34 \sim 7.22(\mathrm{~m}, 4 \mathrm{H}), 7.16 \sim 6.99(\mathrm{~m}, 4 \mathrm{H}), 6.91 \sim 6.78(\mathrm{~m}$, $1 \mathrm{H}), 6.79 \sim 6.70(\mathrm{~m}, 1 \mathrm{H}), 5.93(\mathrm{~d}, J=8.3 \mathrm{~Hz}, 1 \mathrm{H}), 4.34(\mathrm{q}$, $J=8.4 \mathrm{~Hz}, 1 \mathrm{H}), 3.82$ (s, 3H), 3.18 (s, 3H), 3.07 (dd, $J=$ $12.9,8.7 \mathrm{~Hz}, 1 \mathrm{H}), 2.66(\mathrm{dd}, J=12.9,8.2 \mathrm{~Hz}, 1 \mathrm{H}) ;{ }^{13} \mathrm{C} \mathrm{NMR}$ $\left(101 \mathrm{MHz}, \mathrm{CDCl}_{3}\right) \delta: 195.8,174.4,156.3,138.5,136.99$, $136.5,132.7,128.6,128.4,128.2,128.2,127.0,113.2$, $111.8,108.9,85.2,83.9,56.1,48.9,41.9,26.6$; HRMS (ESI) calcd for $\mathrm{C}_{26} \mathrm{H}_{24} \mathrm{NO}_{4}[\mathrm{M}+\mathrm{H}]^{+}$414.1700, found 414.1701.

5-Benzoyl-1'-methyl-4-(4-nitrophenyl)-4,5-dihydro-3 $H$ spiro[furan-2,3'-indolin]-2'-one (4ai'): Yellow solid $(27 \%$ yield), m.p. 135 137 ${ }^{\circ} \mathrm{C}$; ${ }^{1} \mathrm{H}$ NMR (400 MHz, $\left.\mathrm{CDCl}_{3}\right) \delta$ : $7.89 \sim 7.87(\mathrm{~m}, 2 \mathrm{H}), 7.47 \sim 7.44(\mathrm{~m}, 2 \mathrm{H}), 7.36 \sim 7.33(\mathrm{~m}$, $3 \mathrm{H}), 7.29 \sim 7.27(\mathrm{~m}, 1 \mathrm{H}), 7.12 \sim 7.08(\mathrm{~m}, 1 \mathrm{H}), 7.04 \sim 7.03$ $(\mathrm{m}, 1 \mathrm{H}), 6.84(\mathrm{~d}, J=7.8 \mathrm{~Hz}, 1 \mathrm{H}), 6.26(\mathrm{~d}, J=3.3 \mathrm{~Hz}, 1 \mathrm{H})$, $6.11 \sim 6.10(\mathrm{~m}, 1 \mathrm{H}), 5.87(\mathrm{~d}, J=8.5 \mathrm{~Hz}, 1 \mathrm{H}), 4.56 \sim 4.49$ (m, 1H), $3.22(\mathrm{~s}, 3 \mathrm{H}), 3.09$ (dd, $J=12.9,8.7 \mathrm{~Hz}, 1 \mathrm{H}), 2.52$ $(\mathrm{dd}, J=12.9,8.2 \mathrm{~Hz}, 1 \mathrm{H}) ;{ }^{13} \mathrm{C} \mathrm{NMR}\left(101 \mathrm{MHz}, \mathrm{CDCl}_{3}\right) \delta$ : $196.1,174.4,150.7,143.4141 .5,135.8,132.8,130.99$, 
$130.1,129.4,128.9,128.7,128.6,128.1,125.8,123.2$, 123.0, 110.5, 108.6, 107.9, 83.6, 83.1, 42.7, 39.5, 26.5; HRMS (ESI) calcd for $\mathrm{C}_{25} \mathrm{H}_{21} \mathrm{~N}_{2} \mathrm{O}_{5}[\mathrm{M}+\mathrm{H}]^{+} 429.1445$, found 429.1445.

5-Benzoyl-4-(4-fluorophenyl)-1'-methyl-4,5-dihydro3H-spiro[furan-2,3'-indolin]-2'-one (4aj'): White solid (38\% yield), m.p. $220 \sim 222{ }^{\circ} \mathrm{C} ;{ }^{1} \mathrm{H}$ NMR $(400 \mathrm{MHz}$, $\left.\mathrm{CDCl}_{3}\right) \delta: 7.95(\mathrm{~d}, J=7.7 \mathrm{~Hz}, 2 \mathrm{H}), 7.52 \sim 7.42(\mathrm{~m}, 4 \mathrm{H})$, $7.39 \sim 7.26(\mathrm{~m}, 3 \mathrm{H}), 7.08(\mathrm{t}, J=7.5 \mathrm{~Hz}, 1 \mathrm{H}), 7.00(\mathrm{t}, J=8.5$ $\mathrm{Hz}, 2 \mathrm{H}), 6.80$ (d, $J=7.8 \mathrm{~Hz}, 1 \mathrm{H}), 5.71(\mathrm{~d}, J=9.6 \mathrm{~Hz}, 1 \mathrm{H})$, $4.31(\mathrm{dd}, \quad J=20.0,9.5 \mathrm{~Hz}, 1 \mathrm{H}), 3.21(\mathrm{~s}, 3 \mathrm{H}), 2.89 \sim 2.79$ $(\mathrm{m}, 1 \mathrm{H}), 2.66(\mathrm{dd}, J=12.8,8.7 \mathrm{~Hz}, 1 \mathrm{H}) ;{ }^{13} \mathrm{C}$ NMR $(101$ $\left.\mathrm{MHz}, \mathrm{CDCl}_{3}\right) \delta: 195.2,177.1,162.1(\mathrm{~d}, J=244.3 \mathrm{~Hz})$, $143.4,135.5,134.2$ (d, $J=3.2 \mathrm{~Hz}), 133.7,130.1,130.0$, $129.9,129.8,129.5,128.6,124.3,123.5,115.7$ (d, $J=21.2$ Hz), 108.6, 86.6, 83.9, 46.6, 43.96, 26.4; HRMS (ESI) calcd for $\mathrm{C}_{25} \mathrm{H}_{21} \mathrm{FNO}_{3}[\mathrm{M}+\mathrm{H}]^{+}$402.1500, found 402.1503.

5-Benzoyl-4-(4-chlorophenyl)-1'-methyl-4,5-dihydro-3Hspiro[furan-2,3'-indolin]-2'-one (4ak'): White solid (44\% yield), m.p. $221 \sim 223{ }^{\circ} \mathrm{C}$; ${ }^{1} \mathrm{H}$ NMR (400 MHz, $\left.\mathrm{CDCl}_{3}\right) \delta$ : $7.72 \sim 7.71(\mathrm{~m}, 2 \mathrm{H}), 7.50 \sim 7.44(\mathrm{~m}, 2 \mathrm{H}), 7.38 \sim 7.26(\mathrm{~m}$, $5 \mathrm{H}), 7.14 \sim 7.08(\mathrm{~m}, 3 \mathrm{H}), 6.86(\mathrm{~d}, J=7.8 \mathrm{~Hz}, 1 \mathrm{H}), 5.94(\mathrm{~d}$, $J=7.9 \mathrm{~Hz}, 1 \mathrm{H}), 4.31$ (q, $J=7.9 \mathrm{~Hz}, 1 \mathrm{H}), 3.23$ (s, 3H), 2.98 (dd, $J=13.2,7.5 \mathrm{~Hz}, 1 \mathrm{H}), 2.74(\mathrm{dd}, J=13.2,8.5 \mathrm{~Hz}, 1 \mathrm{H})$; ${ }^{13} \mathrm{C}$ NMR (101 MHz, $\left.\mathrm{CDCl}_{3}\right) \delta: 195.5,174.6,143.5,137.5$, $136.4,132.96,132.9,131.4,130.1,130.0,128.4,128.3$, 123.3, 123.1, 108.7, 85.5, 83.5, 48.3, 42.1, 26.5; HRMS (ESI) calcd for $\mathrm{C}_{25} \mathrm{H}_{21} \mathrm{ClNO}_{3}[\mathrm{M}+\mathrm{H}]^{+}$418.1204, found 418.1205.

5-Benzoyl-4-(4-bromophenyl)-1'-methyl-4,5-dihydro-3Hspiro[furan-2,3'-indolin]-2'-one (4al'): White solid (33\% yield), m.p. $180 \sim 182{ }^{\circ} \mathrm{C} ;{ }^{1} \mathrm{H}$ NMR (400 MHz, $\left.\mathrm{CDCl}_{3}\right) \delta$ : $7.69(\mathrm{~d}, J=7.0 \mathrm{~Hz}, 2 \mathrm{H}), 7.49 \sim 7.44(\mathrm{~m}, 2 \mathrm{H}), 7.38 \sim 7.30$ $(\mathrm{m}, 3 \mathrm{H}), 7.24 \sim 7.20(\mathrm{~m}, 4 \mathrm{H}), 7.14 \sim 7.10(\mathrm{~m}, 1 \mathrm{H}), 6.86(\mathrm{~d}$, $J=7.6 \mathrm{~Hz}, 1 \mathrm{H}), 5.96(\mathrm{~d}, J=7.7 \mathrm{~Hz}, 1 \mathrm{H}), 4.31$ (dd, $J=14.8$, $7.2 \mathrm{~Hz}, 1 \mathrm{H}), 3.22(\mathrm{~m}, 3 \mathrm{H}), 3.00 \sim 2.95(\mathrm{~m}, 1 \mathrm{H}), 2.71(\mathrm{dd}$, $J=12.2,8.7 \mathrm{~Hz}, 1 \mathrm{H}) ;{ }^{13} \mathrm{C} \mathrm{NMR}\left(101 \mathrm{MHz}, \mathrm{CDCl}_{3}\right) \delta$ : $195.9,174.9,143.4,137.7,136.2,133.1,131.3,130.3$, 130.2, 128.4, 128.3, 123.3, 123.2, 121.1, 108.8, 85.2, 83.6, 48.4, 42.0, 26.5; HRMS (ESI) calcd for $\mathrm{C}_{25} \mathrm{H}_{21} \mathrm{BrNO}_{3}[\mathrm{M}+$ $\mathrm{H}]^{+}$462.0699, found 462.0700 .

5-Benzoyl-1'-methyl-4-( $p$-tolyl)-4,5-dihydro-3H-spiro[furan-2,3'-indolin]-2'-one (4am'): White solid (38\% yield), m.p. $197 \sim 199{ }^{\circ} \mathrm{C} ;{ }^{1} \mathrm{H}$ NMR $\left(400 \mathrm{MHz}, \mathrm{CDCl}_{3}\right) \delta: 7.72(\mathrm{~d}$, $J=7.7 \mathrm{~Hz}, 2 \mathrm{H}), 7.50(\mathrm{~d}, J=7.3 \mathrm{~Hz}, 1 \mathrm{H}), 7.45 \sim 7.38(\mathrm{~m}$, $1 \mathrm{H}), 7.38 \sim 7.32(\mathrm{~m}, 1 \mathrm{H}), 7.31 \sim 7.26(\mathrm{~m}, 2 \mathrm{H}), 7.22 \sim 7.15$ $(\mathrm{m}, 2 \mathrm{H}), 7.14 \sim 7.07(\mathrm{~m}, 1 \mathrm{H}), 6.88(\mathrm{dd}, J=28.0,7.8 \mathrm{~Hz}$, $3 \mathrm{H}), 5.93$ (d, $J=8.3 \mathrm{~Hz}, 1 \mathrm{H}), 4.36$ (q, $J=8.4 \mathrm{~Hz}, 1 \mathrm{H}), 3.22$ (s, 3H), 3.07 (dd, $J=12.8,9.0 \mathrm{~Hz}, 1 \mathrm{H}), 2.65$ (dd, $J=12.9$, $8.1 \mathrm{~Hz}, 1 \mathrm{H}), 2.19(\mathrm{~s}, 3 \mathrm{H}) ;{ }^{13} \mathrm{C}$ NMR $\left(101 \mathrm{MHz}, \mathrm{CDCl}_{3}\right) \delta$ : $196.3,175.1,143.97,137.2,136.9,135.7,132.8,132.0$, 130.3, 129.2, 128.9, 128.9, 128.4, 123.7, 123.3, 108.9, 85.7, 84.0, 49.1, 42.3, 26.7, 21.2; HRMS (ESI) calcd for $\mathrm{C}_{26} \mathrm{H}_{24^{-}}$ $\mathrm{NO}_{3}[\mathrm{M}+\mathrm{H}]^{+}$398.1751, found 398.1753.

5-Benzoyl-4-(4-methoxyphenyl)-1'-methyl-4,5-dihydro3H-spiro[furan-2,3'-indolin]-2'-one (4an'): White solid (33\% yield), m.p. $189 \sim 191{ }^{\circ} \mathrm{C} ;{ }^{1} \mathrm{H}$ NMR $(600 \mathrm{MHz}$,
$\left.\mathrm{CDCl}_{3}\right) \delta: 7.70(\mathrm{~d}, J=7.4 \mathrm{~Hz}, 2 \mathrm{H}), 7.50(\mathrm{~d}, J=7.1 \mathrm{~Hz}, 1 \mathrm{H})$, $7.45 \sim 7.39(\mathrm{~m}, 1 \mathrm{H}), 7.37 \sim 7.32(\mathrm{~m}, 1 \mathrm{H}), 7.32 \sim 7.27(\mathrm{~m}$, $2 \mathrm{H}), 7.25 \sim 7.20(\mathrm{~m}, 2 \mathrm{H}), 7.11(\mathrm{t}, J=7.5 \mathrm{~Hz}, 1 \mathrm{H}), 6.85(\mathrm{~d}$, $J=7.7 \mathrm{~Hz}, 1 \mathrm{H}), 6.65$ (d, $J=8.6 \mathrm{~Hz}, 2 \mathrm{H}), 5.93$ (d, $J=8.3$ $\mathrm{Hz}, 1 \mathrm{H}), 4.34$ (q, $J=8.3 \mathrm{~Hz}, 1 \mathrm{H}), 3.69$ (s, 3H), 3.23 (s, 3H), $3.03(\mathrm{dd}, J=12.9,8.5 \mathrm{~Hz}, 1 \mathrm{H}), 2.67$ (dd, $J=13.0,8.4 \mathrm{~Hz}$, $1 \mathrm{H}) ;{ }^{13} \mathrm{C} \mathrm{NMR}\left(151 \mathrm{MHz}, \mathrm{CDCl}_{3}\right) \delta: 196.1,174.8,158.5$, $143.5,136.6,132.7,131.6,130.5,129.97,129.7,128.4$, $128.1,123.3,122.97,113.6,108.6,85.3,83.5,55.2,48.3$, 42.2, 26.5; HRMS (ESI) calcd for $\mathrm{C}_{26} \mathrm{H}_{24} \mathrm{NO}_{4}[\mathrm{M}+\mathrm{H}]^{+}$ 414.1700, found 414.1701.

5-Benzoyl-4-(3-bromophenyl)-1'-methyl-4,5-dihydro-3Hspiro[furan-2,3'-indolin]-2'-one (4ao'): White solid (23\% yield), m.p. 206 208 ${ }^{\circ} \mathrm{C}$; ${ }^{1} \mathrm{H}$ NMR (600 MHz, $\left.\mathrm{CDCl}_{3}\right) \delta$ : $7.71(\mathrm{~d}, J=7.4 \mathrm{~Hz}, 2 \mathrm{H}), 7.51 \sim 7.43(\mathrm{~m}, 2 \mathrm{H}), 7.41 \sim 7.29$ $(\mathrm{m}, 5 \mathrm{H}), 7.20(\mathrm{~d}, J=7.8 \mathrm{~Hz}, 1 \mathrm{H}), 7.11(\mathrm{t}, J=7.3 \mathrm{~Hz}, 1 \mathrm{H})$, 7.02 (t, $J=7.8 \mathrm{~Hz}, 1 \mathrm{H}), 6.85$ (d, $J=7.7 \mathrm{~Hz}, 1 \mathrm{H}), 5.94$ (d, $J=7.9 \mathrm{~Hz}, 1 \mathrm{H}), 4.28(\mathrm{q}, J=8.0 \mathrm{~Hz}, 1 \mathrm{H}), 3.22(\mathrm{~s}, 3 \mathrm{H}), 3.01$ (dd, $J=13.0,8.1 \mathrm{~Hz}, 1 \mathrm{H}), 2.70$ (dd, $J=13.1,8.3 \mathrm{~Hz}, 1 \mathrm{H})$; ${ }^{13} \mathrm{C}$ NMR $\left(151 \mathrm{MHz}, \mathrm{CDCl}_{3}\right) \delta: 195.5,174.6,143.5,141.1$, $136.4,132.98,131.8,131.3,130.1,130.1,129.9,128.4$, $128.3,127.2,123.3,123.1,122.1,108.7,85.3,83.5,48.5$, 41.6, 26.5; HRMS (ESI) calcd for $\mathrm{C}_{25} \mathrm{H}_{21} \mathrm{BrNO}_{3}[\mathrm{M}+\mathrm{H}]^{+}$ 462.0699 , found 462.0700 .

5-Benzoyl-4-(2-bromophenyl)-1'-methyl-4,5-dihydro-3Hspiro[furan-2,3'-indolin]-2'-one (4ap'): White solid $(29 \%$ yield), m.p. $203 \sim 205{ }^{\circ} \mathrm{C}$; ${ }^{1} \mathrm{H}$ NMR (600 $\left.\mathrm{MHz} \mathrm{CDCl}_{3}\right) \delta$ : $7.71(\mathrm{~d}, J=7.5 \mathrm{~Hz}, 2 \mathrm{H}), 7.56(\mathrm{~d}, J=7.6 \mathrm{~Hz}, 2 \mathrm{H}), 7.43 \sim$ $7.34(\mathrm{~m}, 2 \mathrm{H}), 7.30 \sim 7.26(\mathrm{~m}, 3 \mathrm{H}), 7.22 \sim 7.11(\mathrm{~m}, 2 \mathrm{H})$, $6.96 \sim 6.90(\mathrm{~m}, 1 \mathrm{H}), 6.86(\mathrm{~d}, J=7.7 \mathrm{~Hz}, 1 \mathrm{H}), 6.16(\mathrm{~d}, J=$ $8.5 \mathrm{~Hz}, 1 \mathrm{H}), 4.89$ (dd, $J=18.4,8.1 \mathrm{~Hz}, 1 \mathrm{H}), 3.23$ (s, 3H), $3.19 \sim 3.13(\mathrm{~m}, 1 \mathrm{H}), 2.52(\mathrm{dd}, J=12.4,7.3 \mathrm{~Hz}, 1 \mathrm{H}) ;{ }^{13} \mathrm{C}$ NMR $\left(151 \mathrm{MHz}, \mathrm{CDCl}_{3}\right) \delta: 196.3,174.7,143.4,136.7$, $136.2,132.8,132.3,131.2,130.1,129.7,128.7,128.3$, $128.2,127.7,125.3,123.4,123.1,108.6,83.4,81.9,47.4$, 40.7, 26.5; HRMS (ESI) calcd for $\mathrm{C}_{25} \mathrm{H}_{21} \mathrm{BrNO}_{3}[\mathrm{M}+\mathrm{H}]^{+}$ 462.0699, found 462.0700 .

5-Benzoyl-1'-methyl-4-(naphthalen-1-yl)-4,5-dihydro-3Hspiro[furan-2,3'-indolin]-2'-one (4aq'): White solid $(38 \%$ yield), m.p. $197 \sim 199{ }^{\circ} \mathrm{C} ;{ }^{1} \mathrm{H}$ NMR (400 MHz, $\left.\mathrm{CDCl}_{3}\right) \delta$ : $7.71 \sim 7.60(\mathrm{~m}, 6 \mathrm{H}), 7.54 \sim 7.39(\mathrm{~m}, 2 \mathrm{H}), 7.35 \sim 7.34(\mathrm{~m}$, 4H), $7.21 \sim 7.12(\mathrm{~m}, 3 \mathrm{H}), 6.87(\mathrm{~d}, J=7.8 \mathrm{~Hz}, 1 \mathrm{H}), 6.04(\mathrm{~d}$, $J=8.2 \mathrm{~Hz}, 1 \mathrm{H}), 4.54(\mathrm{q}, J=8.3 \mathrm{~Hz}, 1 \mathrm{H}), 3.25(\mathrm{~s}, 3 \mathrm{H}), 3.20$ (dd, $J=12.9,8.7 \mathrm{~Hz}, 1 \mathrm{H}), 2.76$ (dd, $J=13.0,8.2 \mathrm{~Hz}, 1 \mathrm{H})$; ${ }^{13} \mathrm{C}$ NMR $\left(101 \mathrm{MHz}, \mathrm{CDCl}_{3}\right) \delta: 195.9,174.8,143.5,136.5$, $135.97,133.1,132.6,132.4,131.5,130.0,128.4,128.1$, $127.97,127.8,127.5,126.6,125.9,125.7,123.3,123.0$, 108.7, 85.3, 83.7, 49.1, 41.9, 26.5; HRMS (ESI) calcd for $\mathrm{C}_{29} \mathrm{H}_{24} \mathrm{NO}_{3}[\mathrm{M}+\mathrm{H}]^{+}$434.1751, found 434.1753.

5-Benzoyl-1'-methyl-4-(naphthalen-2-yl)-4,5-dihydro-3$H$-spiro[furan-2,3'-indolin]-2'-one (4ar'): White solid (26\% yield), m.p. $233 \sim 235{ }^{\circ} \mathrm{C} ;{ }^{1} \mathrm{H}$ NMR (400 MHz, $\left.\mathrm{CDCl}_{3}\right) \delta$ : $7.71 \sim 7.60(\mathrm{~m}, 6 \mathrm{H}), 7.56 \sim 7.49(\mathrm{~m}, 2 \mathrm{H}), 7.38 \sim 7.31(\mathrm{~m}$, $4 \mathrm{H}), 7.21 \sim 7.11(\mathrm{~m}, 3 \mathrm{H}), 6.87(\mathrm{~d}, J=7.8 \mathrm{~Hz}, 1 \mathrm{H}), 6.04(\mathrm{~d}$, $J=8.2 \mathrm{~Hz}, 1 \mathrm{H}), 4.54$ (q, $J=8.4 \mathrm{~Hz}, 1 \mathrm{H}), 3.25$ (s, 3H), 3.20 (dd, $J=12.9,8.7 \mathrm{~Hz}, 1 \mathrm{H}), 2.76(\mathrm{dd}, J=12.9,8.2 \mathrm{~Hz}, 1 \mathrm{H})$; ${ }^{13} \mathrm{C}$ NMR $\left(101 \mathrm{MHz}, \mathrm{CDCl}_{3}\right) \delta: 195.9,174.8,143.5,136.5$, 
$135.99,133.1,132.6,132.4,131.5,130.0,128.4,128.1$, $127.96,127.8,127.5,126.6,125.9,125.7,123.3,123.0$, 108.7, 85.3, 83.7, 49.1, 41.9, 26.5; HRMS (ESI) calcd for $\mathrm{C}_{29} \mathrm{H}_{24} \mathrm{NO}_{3}[\mathrm{M}+\mathrm{H}]^{+}$434.1751, found 434.1753.

5-Benzoyl-4-(furan-2-yl)-1'-methyl-4,5-dihydro-3H-spir o[furan-2,3'-indolin]-2'-one (4as'): White solid (42\% yield), m.p. $207 \sim 209{ }^{\circ} \mathrm{C} ;{ }^{1} \mathrm{H}$ NMR $\left(400 \mathrm{MHz}, \mathrm{CDCl}_{3}\right) \delta: 8.02 \sim$ $8.00(\mathrm{~m}, 2 \mathrm{H}), 7.75(\mathrm{dd}, J=8.3,1.1 \mathrm{~Hz}, 2 \mathrm{H}), 7.58 \sim 7.56(\mathrm{~m}$, $2 \mathrm{H}), 7.50 \sim 7.47(\mathrm{~m}, 2 \mathrm{H}), 7.41 \sim 7.32(\mathrm{~m}, 3 \mathrm{H}), 7.15 \sim 7.11$ $(\mathrm{m}, 1 \mathrm{H}), 6.88$ (d, $J=7.8 \mathrm{~Hz}, 1 \mathrm{H}), 5.98(\mathrm{~d}, J=7.4 \mathrm{~Hz}, 1 \mathrm{H})$, 4.39 (dd, $J=15.6,7.1 \mathrm{~Hz}, 1 \mathrm{H}), 3.23$ (s, 3H), 2.99 (dd, $J=$ 13.3, 6.7 Hz, 1H), $2.85(\mathrm{dd}, J=13.3,8.7 \mathrm{~Hz}, 1 \mathrm{H}) ;{ }^{13} \mathrm{C} \mathrm{NMR}$ $\left(101 \mathrm{MHz} \mathrm{CDCl}_{3}\right) \delta: 194.8,174.6,147.3,146.8,143.6$, $135.96,133.4,131.1,130.4,129.6,128.51,128.45,123.4$, 123.3, 123.2, 108.9, 85.8, 83.5, 48.5, 41.9, 26.5; HRMS (ESI) calcd for $\mathrm{C}_{23} \mathrm{H}_{20} \mathrm{NO}_{4}[\mathrm{M}+\mathrm{H}]^{+}$374.1387, found 374.1388 .

5-(4-Fluorobenzoyl)-1'-methyl-4-phenyl-4,5-dihydro-3 $H$ spiro[furan-2,3'-indolin]-2'-one (4ba'): White solid (40\% yield), m.p. $197 \sim 199{ }^{\circ} \mathrm{C}$; ${ }^{1} \mathrm{H}$ NMR $\left(400 \mathrm{MHz}, \mathrm{CDCl}_{3}\right) \delta$ : $7.81 \sim 7.77(\mathrm{~m}, 2 \mathrm{H}), 7.50(\mathrm{~d}, J=7.2 \mathrm{~Hz}, 1 \mathrm{H}), 7.38 \sim 7.31$ $(\mathrm{m}, 3 \mathrm{H}), 7.15 \sim 7.06(\mathrm{~m}, 4 \mathrm{H}), 6.97 \sim 6.92(\mathrm{~m}, 2 \mathrm{H}), 6.86(\mathrm{~d}$, $J=7.8 \mathrm{~Hz}, 1 \mathrm{H}), 5.88(\mathrm{~d}, J=8.3 \mathrm{~Hz}, 1 \mathrm{H}), 4.38(\mathrm{q}, J=8.4$ $\mathrm{Hz}, 1 \mathrm{H}), 3.23$ (s, 3H), 3.09 (dd, $J=12.9,8.9 \mathrm{~Hz}, 1 \mathrm{H})$, $2.73 \sim 2.61(\mathrm{~m}, 1 \mathrm{H}) ;{ }^{13} \mathrm{C} \mathrm{NMR}\left(101 \mathrm{MHz}, \mathrm{CDCl}_{3}\right) \delta: 194.7$, $174.8,165.4(\mathrm{~d}, J=253.2 \mathrm{~Hz}), 143.5,138.2,132.93(\mathrm{~d}, J=$ $3.1 \mathrm{~Hz}), 131.3$ (d, $J=9.3 \mathrm{~Hz}), 131.33,130.1,128.6,128.3$, 127.1, 123.3, 123.1, 115.2 (d, $J=21.8 \mathrm{~Hz}), 108.7$, 85.5, 83.8, 48.97, 41.5, 26.5; HRMS (ESI) calcd for $\mathrm{C}_{25} \mathrm{H}_{21} \mathrm{FN}-$ $\mathrm{O}_{3}[\mathrm{M}+\mathrm{H}]^{+} 402.1500$, found 402.1503 .

5-(4-chlorobenzoyl)-1'-methyl-4-phenyl-4,5-dihydro-3Hspiro[furan-2,3'-indolin]-2'-one (4ca'): White solid (44\% yield), m.p. $178 \sim 180{ }^{\circ} \mathrm{C} ;{ }^{1} \mathrm{H}$ NMR $\left(400 \mathrm{MHz}, \mathrm{CDCl}_{3}\right) \delta$ : $7.69(\mathrm{~d}, J=8.6 \mathrm{~Hz}, 2 \mathrm{H}), 7.51 \sim 7.48(\mathrm{~m}, 1 \mathrm{H}), 7.39 \sim 7.30$ $(\mathrm{m}, 3 \mathrm{H}), 7.27 \sim 7.23(\mathrm{~m}, 2 \mathrm{H}), 7.17 \sim 7.08(\mathrm{~m}, 4 \mathrm{H}), 6.86(\mathrm{~d}$, $J=7.8 \mathrm{~Hz}, 1 \mathrm{H}), 5.87(\mathrm{t}, J=6.8 \mathrm{~Hz}, 1 \mathrm{H}), 4.49 \sim 4.27(\mathrm{~m}$, $1 \mathrm{H}), 3.23$ (s, 3H), 3.08 (dd, $J=12.9,9.0 \mathrm{~Hz}, 1 \mathrm{H}), 2.67$ (dd, $J=12.9,8.2 \mathrm{~Hz}, 1 \mathrm{H}) ;{ }^{13} \mathrm{C} \mathrm{NMR}\left(101 \mathrm{MHz}, \mathrm{CDCl}_{3}\right) \delta$ : $195.2,174.8,143.4,139.1,138.1,134.8,131.3,130.1$, $130.0,129.8,129.6,129.4,129.1,128.6,128.4,128.3$, 127.2, 123.3, 123.1, 108.7, 85.5, 83.8, 48.95, 41.5, 26.5; HRMS (ESI) calcd for $\mathrm{C}_{25} \mathrm{H}_{21} \mathrm{ClNO}_{3}[\mathrm{M}+\mathrm{H}]^{+} 418.1204$, found 418.1205 .

5-(4-Bromobenzoyl)-1'-methyl-4-phenyl-4,5-dihydro-3Hspiro[furan-2,3'-indolin]-2'-one (4da'): White solid (36\% yield), m.p. $218 \sim 220{ }^{\circ} \mathrm{C}$; ${ }^{1} \mathrm{H}$ NMR (400 MHz, $\left.\mathrm{CDCl}_{3}\right) \delta$ : $7.60(\mathrm{t}, J=5.4 \mathrm{~Hz}, 2 \mathrm{H}), 7.49(\mathrm{~d}, J=7.2 \mathrm{~Hz}, 1 \mathrm{H}), 7.45 \sim 7.28$ $(\mathrm{m}, 5 \mathrm{H}), 7.19 \sim 7.04(\mathrm{~m}, 4 \mathrm{H}), 6.86(\mathrm{~d}, J=7.8 \mathrm{~Hz}, 1 \mathrm{H}), 5.86$ $(\mathrm{d}, J=8.3 \mathrm{~Hz}, 1 \mathrm{H}), 4.38$ (q, $J=8.4 \mathrm{~Hz}, 1 \mathrm{H}), 3.23$ (s, 3H), $3.08(\mathrm{dd}, J=12.9,9.0 \mathrm{~Hz}, 1 \mathrm{H}), 2.67(\mathrm{dd}, J=12.9,8.2 \mathrm{~Hz}$, $1 \mathrm{H}) ;{ }^{13} \mathrm{C} \mathrm{NMR}\left(101 \mathrm{MHz}, \mathrm{CDCl}_{3}\right) \delta: 195.4,174.7,143.5$, $138.1,135.2,132.6,131.4,131.3,130.1,130.1,129.8$, $128.6,128.3,127.9,127.2,123.3,123.1,108.7,85.5,83.8$, $48.9,41.5,26.5$; HRMS (ESI) calcd for $\mathrm{C}_{25} \mathrm{H}_{21} \mathrm{BrNO}_{3}[\mathrm{M}+$ $\mathrm{H}]^{+}$462.0699, found 462.0700 .

1'-Methyl-5-(4-methylbenzoyl)-4-phenyl-4,5-dihydro-3Hspiro[furan-2,3'-indolin]-2'-one (4ea'): White solid (42\% yield), m.p. $240 \sim 242{ }^{\circ} \mathrm{C}$; ${ }^{1} \mathrm{H}$ NMR (400 MHz, $\left.\mathrm{CDCl}_{3}\right) \delta$ : $7.63(\mathrm{~d}, J=8.2 \mathrm{~Hz}, 2 \mathrm{H}), 7.54 \sim 7.46(\mathrm{~m}, 1 \mathrm{H}), 7.38 \sim 7.30$ $(\mathrm{m}, 3 \mathrm{H}), 7.16 \sim 7.03(\mathrm{~m}, 6 \mathrm{H}), 6.85(\mathrm{~d}, J=7.8 \mathrm{~Hz}, 1 \mathrm{H}), 5.93$ $(\mathrm{d}, J=8.2 \mathrm{~Hz}, 1 \mathrm{H}), 4.37$ (q, $J=8.4 \mathrm{~Hz}, 1 \mathrm{H}), 3.23(\mathrm{~s}, 3 \mathrm{H})$, $3.14 \sim 3.03(\mathrm{~m}, 1 \mathrm{H}), 2.68(\mathrm{dt}, J=23.0,11.5 \mathrm{~Hz}, 1 \mathrm{H}), 2.32$ $(\mathrm{s}, 3 \mathrm{H}) ;{ }^{13} \mathrm{C} \mathrm{NMR}\left(101 \mathrm{MHz}, \mathrm{CDCl}_{3}\right) \delta: 195.4,174.7,143.5$, $143.5,138.6,133.99,131.5,129.97,128.9,128.6,128.6$, $128.2,126.99,123.3,122.96,108.6,85.1,83.5,48.99$, 41.96, 26.5, 21.6; HRMS (ESI) calcd for $\mathrm{C}_{26} \mathrm{H}_{24} \mathrm{NO}_{3}[\mathrm{M}+$ $\mathrm{H}]^{+}$398.1751, found 398.1753.

5-(4-Methoxybenzoyl)-1'-methyl-4-phenyl-4,5-dihydro3H-spiro[furan-2,3'-indolin]-2'-one (4fa'): White solid (29\% yield), m.p. $205 \sim 207{ }^{\circ} \mathrm{C} ;{ }^{1} \mathrm{H}$ NMR (400 MHz, $\left.\mathrm{CDCl}_{3}\right) \delta: 7.74 \sim 7.68(\mathrm{~m}, 2 \mathrm{H}), 7.50(\mathrm{~d}, J=6.9 \mathrm{~Hz}, 1 \mathrm{H})$, $7.45 \sim 7.39(\mathrm{~m}, 1 \mathrm{H}), 7.38 \sim 7.32(\mathrm{~m}, 1 \mathrm{H}), 7.32 \sim 7.26(\mathrm{~m}$, $2 \mathrm{H}), 7.25 \sim 7.21(\mathrm{~m}, 2 \mathrm{H}), 7.11(\mathrm{dd}, J=11.0,4.0 \mathrm{~Hz}, 1 \mathrm{H})$, $6.85(\mathrm{~d}, J=7.8 \mathrm{~Hz}, 1 \mathrm{H}), 6.68 \sim 6.60(\mathrm{~m}, 2 \mathrm{H}), 5.93(\mathrm{~d}, J=$ $8.2 \mathrm{~Hz}, 1 \mathrm{H}), 4.34$ (q, $J=8.3 \mathrm{~Hz}, 1 \mathrm{H}), 3.68(\mathrm{~s}, 3 \mathrm{H}), 3.23$ (s, $3 \mathrm{H}), 3.02$ (dd, $J=13.0,8.4 \mathrm{~Hz}, 1 \mathrm{H}), 2.68$ (dd, $J=13.0,8.3$ $\mathrm{Hz}, 1 \mathrm{H}) ;{ }^{13} \mathrm{C}$ NMR $\left(101 \mathrm{MHz}, \mathrm{CDCl}_{3}\right) \delta: 196.1,174.7$, $158.5,143.5,136.5,132.7,131.5,130.5,129.98,129.7$, $128.4,128.2,123.3,122.98,113.6,108.6,85.3,83.5,55.2$, 48.3, 42.2, 26.5; HRMS (ESI) calcd for $\mathrm{C}_{26} \mathrm{H}_{24} \mathrm{NO}_{4}[\mathrm{M}+$ $\mathrm{H}]^{+}$414.1700, found 414.1701.

5-(3-Bromobenzoyl)-1'-methyl-4-phenyl-4,5-dihydro-3Hspiro[furan-2,3'-indolin]-2'-one (4ga'): White solid (42\% yield), m.p. 219 221 ${ }^{\circ} \mathrm{C}$; ${ }^{1} \mathrm{H}$ NMR (400 MHz, $\left.\mathrm{CDCl}_{3}\right) \delta$ : $7.77 \sim 7.72(\mathrm{~m}, 1 \mathrm{H}), 7.67(\mathrm{~d}, J=7.8 \mathrm{~Hz}, 1 \mathrm{H}), 7.55 \sim 7.47$ $(\mathrm{m}, 2 \mathrm{H}), 7.39 \sim 7.33(\mathrm{~m}, 1 \mathrm{H}), 7.32 \sim 7.27(\mathrm{~m}, 2 \mathrm{H}), 7.19 \sim$ $7.06(\mathrm{~m}, 5 \mathrm{H}), 6.86(\mathrm{~d}, J=7.8 \mathrm{~Hz}, 1 \mathrm{H}), 5.88(\mathrm{~d}, J=8.3 \mathrm{~Hz}$, $1 \mathrm{H}), 4.45 \sim 4.32(\mathrm{~m}, 1 \mathrm{H}), 3.23(\mathrm{~s}, 3 \mathrm{H}), 3.08(\mathrm{dt}, J=13.0,6.5$ $\mathrm{Hz}, 1 \mathrm{H}), 2.66(\mathrm{dd}, J=12.9,8.1 \mathrm{~Hz}, 1 \mathrm{H}) ;{ }^{13} \mathrm{C}$ NMR $(101$ $\left.\mathrm{MHz}, \mathrm{CDCl}_{3}\right) \delta: 194.9,174.7,143.4,138.2,137.9,135.5$, $131.4,131.2,130.1,129.7,128.5,128.4,127.3,127.0$, 123.3, 123.1, 122.4, 108.7, 85.1, 83.8, 48.9, 41.4, 26.5; HRMS (ESI) calcd for $\mathrm{C}_{25} \mathrm{H}_{21} \mathrm{BrNO}_{3}[\mathrm{M}+\mathrm{H}]^{+}$462.0699, found 462.0700 .

5-(3-Methoxybenzoyl)-1'-methyl-4-phenyl-4,5-dihydro$3 H$-spiro[furan-2,3'-indolin]-2'-one (4ha'): White solid (37\% yield), m.p. $170 \sim 172{ }^{\circ} \mathrm{C} ;{ }^{1} \mathrm{H}$ NMR (400 MHz, $\left.\mathrm{CDCl}_{3}\right) \delta: 7.53 \sim 7.47(\mathrm{~m}, 1 \mathrm{H}), 7.40 \sim 7.28(\mathrm{~m}, 4 \mathrm{H}), 7.25 \sim$ $7.04(\mathrm{~m}, 6 \mathrm{H}), 7.03 \sim 6.93(\mathrm{~m}, 1 \mathrm{H}), 6.91 \sim 6.78(\mathrm{~m}, 1 \mathrm{H}), 5.84$ $(\mathrm{dd}, J=87.2,8.9 \mathrm{~Hz}, 1 \mathrm{H}), 4.44 \sim 4.23(\mathrm{~m}, 1 \mathrm{H}), 3.74(\mathrm{~d}, J=$ $6.7 \mathrm{~Hz}, 3 \mathrm{H}), 3.23$ (s, 3H), 3.09 (dd, $J=12.9,8.7 \mathrm{~Hz}, 1 \mathrm{H})$, $2.69(\mathrm{dd}, J=12.9,8.2 \mathrm{~Hz}, 1 \mathrm{H}) ;{ }^{13} \mathrm{C}$ NMR $(101 \mathrm{MHz}$, $\left.\mathrm{CDCl}_{3}\right) \delta: 195.8,174.7,159.4,143.5,138.5,137.9,131.4$, $130.0,129.1,128.8,128.7,128.2,127.0,123.3,123.0$, $120.9,119.6,112.4,108.7,85.2,83.6,55.4,48.99,41.8$, 26.5; HRMS (ESI) calcd for $\mathrm{C}_{26} \mathrm{H}_{24} \mathrm{NO}_{4}[\mathrm{M}+\mathrm{H}]{ }^{+}$ 414.1700, found 414.1701.

5-(2-Methoxybenzoyl)-1'-methyl-4-phenyl-4,5-dihydro3H-spiro[furan-2,3'-indolin]-2'-one (4ia'): White solid (29\% yield), m.p. $158 \sim 160{ }^{\circ} \mathrm{C} ;{ }^{1} \mathrm{H}$ NMR (400 MHz, $\left.\mathrm{CDCl}_{3}\right) \delta: 7.47(\mathrm{~d}, J=7.1 \mathrm{~Hz}, 1 \mathrm{H}), 7.38 \sim 7.23(\mathrm{~m}, 4 \mathrm{H})$, $7.15 \sim 6.99(\mathrm{~m}, 5 \mathrm{H}), 6.89 \sim 6.76(\mathrm{~m}, 2 \mathrm{H}), 6.76 \sim 6.68(\mathrm{~m}$, $1 \mathrm{H}), 6.16(\mathrm{~d}, J=8.2 \mathrm{~Hz}, 1 \mathrm{H}), 4.27$ (q, $J=8.2 \mathrm{~Hz}, 1 \mathrm{H}), 3.90$ $(\mathrm{d}, J=8.9 \mathrm{~Hz}, 3 \mathrm{H}), 3.23$ (s, 3H), 2.94 (dd, $J=13.0,7.9 \mathrm{~Hz}$, 
$1 \mathrm{H}), 2.67(\mathrm{dd}, J=13.0,8.6 \mathrm{~Hz}, 1 \mathrm{H}) ;{ }^{13} \mathrm{C} \mathrm{NMR}(101 \mathrm{MHz}$, $\left.\mathrm{CDCl}_{3}\right) \delta: 196.9,174.7,157.3,143.6,139.9,133.3,131.8$, $130.8,129.8,128.5,128.0,127.2,126.8,123.3,122.8$, $120.8,110.8,108.6,88.1,82.8,55.7,47.8,42.9,26.4$; HRMS (ESI) calcd for $\mathrm{C}_{26} \mathrm{H}_{24} \mathrm{NO}_{4}[\mathrm{M}+\mathrm{H}]^{+} 414.1700$, found 414.1701.

\subsection{General procedure for the synthesis of $5^{\prime}$}

Hydroxylamine hydrochloride $(106 \mathrm{mg}, 1.5 \mathrm{mmol})$ and sodium acetate $(100 \mathrm{mg}, 1.2 \mathrm{mmol})$ were added to a solution of 4al' (92 $\mathrm{mg}, 0.20 \mathrm{mmol})$ in methanol $(5 \mathrm{~mL})$. Then, the reaction mixture was refluxed in a heating mantle for $10 \mathrm{~h}$. After cooling to room temperature, water $(3 \mathrm{~mL})$ was added to quench the reaction. The solution was extracted with $\mathrm{CH}_{2} \mathrm{Cl}_{2}(10 \mathrm{~mL} \times 3)$. The combined organic layer was washed with brine, dried over $\mathrm{Na}_{2} \mathrm{SO}_{4}$ and concentrated under reduced pressure. The crude reaction mixture was purified via column chromatography on silica gel to afford pure product $\mathbf{5}^{\prime}$ as colorless solid in $96 \%$ yield.

4-(4-Bromophenyl)-5-((E)-(hydroxyimino)(phenyl)methyl)-1'-methyl-4,5-dihydro-3H-spiro[furan-2,3'-indolin]$2^{\prime}$-one (5'): White solid (96\% yield), m.p. $183 \sim 184{ }^{\circ} \mathrm{C} ;{ }^{1} \mathrm{H}$ NMR (400 MHz, DMSO- $\left.d_{6}\right) \delta: 11.71(\mathrm{~s}, 1 \mathrm{H}), 7.65(\mathrm{~d}, J=$ $7.2 \mathrm{~Hz}, 1 \mathrm{H}), 7.52(\mathrm{~d}, J=8.5 \mathrm{~Hz}, 2 \mathrm{H}), 7.41 \sim 7.36(\mathrm{~m}, 3 \mathrm{H})$, $7.26 \sim 7.22(\mathrm{~m}, 1 \mathrm{H}), 7.17 \sim 7.11(\mathrm{~m}, 5 \mathrm{H}), 7.07(\mathrm{~d}, J=7.8$ $\mathrm{Hz}, 1 \mathrm{H}), 5.92(\mathrm{~d}, J=7.2 \mathrm{~Hz}, 1 \mathrm{H}), 4.54 \sim 4.49(\mathrm{~m}, 1 \mathrm{H}), 3.17$ (s, 3H), 2.82 (dd, $J=13.7,9.6 \mathrm{~Hz}, 1 \mathrm{H}), 2.58(\mathrm{dd}, J=13.7$, $4.7 \mathrm{~Hz}, 1 \mathrm{H}), 2.03 \sim 1.96(\mathrm{~m}, 1 \mathrm{H}) ;{ }^{13} \mathrm{C} \mathrm{NMR}(101 \mathrm{MHz}$, DMSO) $\delta$ : 174.8, 156.4, 143.5, 141.7, 134.5, 131.95, 131.5, $130.8,130.3,130.1,129.0,128.6,127.6,124.3,123.5$, 120.2, 109.6, 82.6, 81.4, 46.5, 41.96, 26.7; HRMS (ESI) calcd for $\mathrm{C}_{25} \mathrm{H}_{22} \mathrm{BrN}_{2} \mathrm{O}_{3}[\mathrm{M}+\mathrm{H}]^{+}$477.0808, found 477.0809 .

Supporting information ${ }^{1} \mathrm{H}$ NMR and ${ }^{13} \mathrm{C}$ NMR spectra of $4^{\prime}$ and $5^{\prime}$. The Supporting Information is available free of charge via the internet at http://sioc-journal.cn/.

\section{References and note}

[1] (a) Rottmann, M.; McNamara, C.; Yeung, B. K. S.; Lee, M. C. S.; Zou, B.; Russell, B.; Seitz, P.; Plouffe, D. M.; Dharia, N. V.; Tan, J.; Cohen, S. B.; Spencer, K. R.; Gonzalez-Paez, G. E.; Lakshminarayana, S. B.; Goh, A.; Suwanarusk, R.; Jegla, T.; Schmitt, E. K.; Beck, H. P.; Brun, R.; Nosten, F.; Renia, L.; Dartois, V.; Keller, T. H.; Fidock, D. A.; Winzeler, E. A.; Diagana, T. T. Science 2010, 329, 1175.

(b) Singh, G. S.; Desta, Z. Y. Chem. Rev. 2012, 112, 6104.

(c) Zheng, Y. J.; Tice, C. M.; Singh, S. B. Bioorg. Med. Chem. Lett. 2014, 24, 3673.

(d) Yu, B.; Yu, D. Q.; Liu, H. M. Eur. J. Med. Chem. 2015, 97, 673.

(e) Ye, N.; Chen, H.; Wold, E. A.; Shi, P.-Y.; Zhou, J. ACS Infect. Dis. 2016, 2, 382 .

(f) Mei, G. J.; Shi, F. Chem. Commun. 2018, 54, 6607.

[2] (a) Peddibhotla, S. Curr. Bioact. Compd. 2009, 5, 20.

(b) Cui, B.-D.; Zuo, J.; Zhao, J.-Q.; Zhou, M.-Q.; Wu, Z.-J.; Zhang, X.-M.; Yuan, W.-C. J. Org. Chem. 2014, 79, 5305.

[3] (a) Vintonyak, V. V.; Warburg, K.; Kruse, H.; Grimme, S.; Hübel, K.; Rauh, D.; Waldmann, H. Angew. Chem., Int. Ed. 2010, 49, 5902.

(b) Jiang, X.; Cao, Y.; Wang, Y.; Liu, L.; Shen, F.; Wang, R. J. Am.
Chem. Soc. 2010, 132, 15328.

(c) Crosignani, S.; Jorand-Lebrun, C.; Page, P.; Campbell, G.; Colovray, V.; Missotten, M.; Humbert, Y.; Cleva, C.; Arrighi, J.-F.; Gaudet, M.; Johnson, Z.; Ferro, P.; Chollet, A. ACS Med. Chem. Lett. 2011, 2, 644.

(d) Rana, S.; Natarajan, A. Org. Biomol. Chem. 2013, 11, 244.

[4] Franz, A. K.; Dreyfuss, P. D.; Schreiber, S. L. J. Am. Chem. Soc. 2007, 129, 1020.

[5] Ghosh, A.; Carter, R. G. Angew. Chem., Int. Ed. 2019, 58, 681.

[6] (a) Alcaide, B.; Almendros, P.; Rodriguez-Acebes, R. J. Org. Chem. 2006, 71, 2346.

(b) Li, J.; Liu, Y. J.; Li, C. J.; Jia, X. Chem.-Eur. J. 2011, 17, 7409.

(c) Hanhan, N. V.; Jones, N. R.; Tran, N. T.; Franz, A. K. Angew. Chem., Int. Ed. 2012, 51, 989.

(d) Yang, L.; Xie, P.; Li, E.; Li, X.; Huang, Y.; Chen, R. Org. Biomol. Chem. 2012, 10, 7628.

(e) Lian, Z.; Shi, M. Eur. J. Org. Chem. 2012, 2012, 581.

(f) Mei, L.-Y.; Wei, Y.; Xu, Q.; Shi, M. Organometallics 2013, 32, 3544 .

(g) Tang, Z.; Liu, Z.; An, Y.; Jiang, R.; Zhang, X.; Li, C.; Jia, X.; Li, J. J. Org. Chem. 2016, 81, 9158.

(h) Silvi, M.; Chatterjee, I.; Liu, Y.-K.; Melchiorre, P. Angew. Chem., Int. Ed. 2013, 52, 10780.

(i) Zhao, B.-L.; Du, D.-M. Adv. Synth. Catal. 2016, 358, 3992.

(j) Zhu, Y.-S.; Wang, W.-B.; Yuan, B.-B.; Li, Y.-N.; Wang, Q.-L.; Bu, Z.-W. Org. Biomol. Chem. 2017, 15, 984.

(k) Zhang, J.-H.; Wang, R.-B.; Li, D.-F.; Zhao, L.-M. ACS Omega 2017, 2, 7022 .

[7] (a) Lee, S.; Hartwig, J. F. J. Org. Chem. 2001, 66, 3402. (b) Jones, K.; Wilkinson, J. Chem. Commun. 1992, 1767.

(c) Overman, L. E.; Rosen, M. D. Angew. Chem., Int. Ed. 2000, 39, 4596.

(d) Kyei, A. S.; Tchabanenko, K.; Baldwin, J. E.; Adlington, R. M. Tetrahedron Lett. 2004, 45, 8931.

(e) Jaegli, S.; Dufour, J.; Wei, H.-L.; Piou, T.; Duan, X.-H.; Vors, J.-P.; Neuville, L.; Zhu, J.-P. Org. Lett. 2010, 12, 4498.

(f) Yin, B.-L.; Lai, J.-Q.; Zhang, Z.-R.; Jiang, H.-F. Adv. Synth. Catal. 2011, 353, 1961.

[8] (a) Hua, Y.-Z.; Lu, L.-J.; Huang, P.-J.; Wei, D.-H.; Tang, M.-S.; Wang, M.-C.; Chang, J.-B. Chem.-Eur. J. 2014, 20, 12394.

(b) Hua, Y.-Z.; Han, X.-W.; Yang, X.-C.; Song, X.; Wang, M.-C.; Chang, J.-B. J. Org. Chem. 2014, 79, 11690.

(c) Hua, Y.-Z.; Yang, X.-C.; Liu, M.-M.; Song, X.; Wang, M.-C.; Chang, J.-B. Macromolecules 2015, 48, 1651.

(e) Wang, X.-W.; Hua, Y.-Z.; Wang, M.-C. J. Org. Chem. 2016, 81, 9227.

(f) Shao, N.; Luo, Y.-Y.; Lu, H.-J.; Hua, Y.-Z.; Wang, M.-C. Tetrahedron 2018, 74, 2130.

(g) Hua, Y.-Z.; Han, X.-W.; Huang, L.-H.; Wang, M.-C. Chin. J. Org. Chem. 2018, 38, 237 (in Chinese).

(华远照, 韩兴旺, 黄利华, 王敏灿, 有机化学, 2018, 38, 237.)

(h) Hua, Y.-Z.; Chen, J.-W.; Yang, H.; Wang, M.-C. J. Org. Chem. 2018, 83, 1160 .

(i) Zhang, Z.-F.; Yang, X.-C.; Lu, H.-J.; Wang, M.-C. Eur. J. Org. Chem. 2018, 785.

(j) Gao, Y.-Y.; Hua, Y.-Z.; Wang, M.-C. Adv. Synth. Catal. 2018, 360,80 .

(k) Liu, S.; Gao, W.-C.; Miao, Y.-H.; Wang, M.-C. J. Org. Chem. 2019, 84, 2652.

[9] (a) Song, X.; Liu, J.; Liu, M.-M.; Wang, X.; Zhang, Z.-F.; Wang, M.-C.; Chang, J. Tetrahedron 2014, 70, 5468.

(b) Hua, Y.-Z.; Liu, M.-M.; Huang, P.-J.; Song, X.; Wang, M.-C.; Chang, J.-B. Chem.-Eur. J. 2015, 21, 11994.

(c) Tao, B.-K.; Yang, H.; Hua, Y.-Z.; Wang, M.-C. Org. Biomol. Chem. 2019, 17, 4301.

(d) Miao, Y.-H.; Hua, Y.-Z.; Wang, M.-C. Org. Biomol. Chem. 2019, 17, 7172 .

(e) Liu, M.-M.; Yang, X.-C.; Hua, Y.-Z.; Chang, J.-B.; Wang, M.-C. 
Org. Lett. 2019, 21, 7089.

(f) Yi, Y.; Hua, Y.-Z.; Lu, H.-J.; Liu, L.-T.; Wang, M.-C. Org. Lett. $2020,22,2527$.

(g) Liu, M.-M.; Yang, X.-C.; Hua, Y.-Z.; Chang, J.-B.; Wang, M.-C. Org. Lett. 2019, 21, 2111.

(h) Yang, X.-C.; Liu, M.-M.; Mathey, F.; Yang, H.; Hua, Y.-Z.; Wang, M.-C. J. Org. Chem. 2019, 84, 7762.
[10] Guo, Y.-J.; Guo, X.; Kong, D.-Z.; Lu, H.-J.; Liu, L.-T.; Hua, Y.-Z.; Wang, M.-C. J. Org. Chem., 2020, 85, 4195. The minor diastereomers in this article are obtained in $23 \% \sim 44 \%$ isolated yields with about $1: 1 d r$ value in the current study.

[11] CCDC $1987133\left(5^{\prime}\right)$ contains the supplementary crystallographic data for this paper. These data can be obtained free of charge from the Cambridge Crystallographic Data Centre.

(Li, L.; Fan, Y.) 